\title{
Rotating and modulated rotating waves in transitions of an enclosed swirling flow
}

\author{
By J. M. LOPEZ \\ Department of Mathematics and Statistics, Arizona State University, Tempe, AZ 85287, USA
}

(Received 23 June 2005 and in revised form 30 September 2005)

The transitions of the flow in an enclosed cylinder driven by the constant rotation of an endwall, from steady axisymmetric flow to aperiodic flow characterized by intermittent bursting dynamics where all the spatial and spatio-temporal symmetries have been broken, is studied numerically. The problem is controlled by two parameters, the Reynolds number and the cylinder aspect ratio. We vary the Reynolds number, fixing the aspect ratio at a value where the primary bifurcation of the axisymmetric steady state is to an axisymmetric periodic flow. The final transition to weak turbulence, however, is governed by a non-axisymmetric branch of rotating waves, which is the primary mode at lower aspect ratios, and the various branches of modulated rotating waves associated with subsequent bifurcations from the rotating wave. We study in detail the spatio-temporal characteristics of the various states encountered along the way, and how the symmetry of the problem impacts on the transition dynamics.

\section{Introduction}

Swirling flows in cylinders driven by the rotation of one or both endwalls are not only of fundamental interest, but also of wide practical interest. These flows provide prototypical laboratory scale models of geophysical flows (Read 2001) and industrial devices including rotating compressors, turbine disks, and computer hard drives. Of fundamental interest in all of these applications is an identification and characterization of the transition processes from laminar flow to turbulence. Despite intensive efforts, a full understanding of the transition process in swirling flows is still lacking.

Low-dimensional dynamical systems theory has provided much guidance and understanding on the transition processes from steady laminar flow to turbulence, inspired by works such as Ruelle \& Takens (1971). Most studies that have focused on relating low-dimensional dynamical systems theory to transitions in fluid flows have been in geometrically simple flows, where the problem is well-defined, boundary conditions well-known, and both laboratory experiments and numerical simulations can be performed with a high degree of precision. The geometric simplicity of these problems is associated with symmetries, and it is well-known that symmetries can lead to dynamical behaviour which would be unexpected (i.e. degenerate) in dynamical systems in the absence of symmetries. The transitions to complex dynamics typically found in very-low-dimensional dynamical systems, e.g. period-doubling cascades (Feigenbaum 1978) and Ruelle-Takens-Newhouse scenarios (Newhouse, Ruelle \& Takens 1978), are either modified or destroyed and replaced by others when the dynamical systems have symmetries. For example, when a system has reflection symmetry, period-doubling is inhibited (Swift \& Wiesenfeld 1984). There have been 
substantial developments in our understanding of dynamical systems with symmetries (e.g. Golubitsky, Stewart \& Schaeffer 1988; Crawford \& Knobloch 1991; Chossat \& Iooss 1994; Chossat \& Lauterbach 2000; Golubitsky \& Stewart 2002). Symmetries typically lead to a large multiplicity of co-existing states (Coles 1965; Mullin 1999), and if the system is governed by a large number of parameters, complicated dynamics associated with high codimensional behaviour is very common. In order to reduce these sources of complications, in this paper, we study the transitions to complex flow in a geometrically very simple flow with minimal symmetry and a minimal number of control (bifurcation) parameters.

The flow under investigation is of an incompressible fluid in a completely filled stationary circular cylinder, of length-to-radius aspect ratio $\Gamma=H / R$, driven by the constant rotation $\left(\Omega \mathrm{rad} \mathrm{s}^{-1}\right)$ of one of its endwalls. The system has $S O(2)$ symmetry, invariance to rotations about the axis, and is governed by only two parameters, $\Gamma$ and the Reynolds number $R e=\Omega R^{2} / v$, where $v$ is the kinematic viscosity. There has been considerable interest in the limit of small $\Gamma$ and where the two endwalls can rotate independently (e.g. Schouveiler et al. 1999; Gauthier, Gondret \& Rabaud 1999; Gauthier et al. 2002; Serre, Tuliszka-Sznitko \& Bontoux 2004); in these cases the transition process is dominated by the instability of the boundary layers on the endwalls (which are thought of as disks), which results in spiral modes of very large azimuthal wavenumber. The case of independently rotating endwalls introduces new phenomena associated with internal shear layers (Lopez 1998; Lopez et al. 2002a; Moisy, Pasutto \& Rabaud 2003) and in the exactly counter-rotating endwalls case, near-heteroclinic behaviour associated with the extra symmetry has been reported (Nore et al. 2003; Nore, Moisy \& Quartier 2005). In the present study, we shall focus on moderate values of $\Gamma$ and a single rotating endwall where the transition process is dominated by the internal redistribution of angular momentum. For sufficiently large rotations of the endwall, i.e. sufficiently large Reynolds number, a meridional flow is established that advects angular momentum in toward the axis so as to establish a central vortex flow. For a range of $\operatorname{Re}$ and $\Gamma$, this central vortex has steady axisymmetric recirculation zones, commonly referred to as vortex breakdown bubbles (Escudier 1984). This visually spectacular aspect of the basic state of this flow has lead to many studies of it features (e.g. Lugt \& Abboud 1987; Lopez 1990; Spohn, Mory \& Hopfinger 1998; Brons, Voigt \& Sorensen 1999; Sotiropoulos \& Ventikos 2001; Sotiropoulos, Ventikos \& Lackey 2001; Ventikos 2002).

There have been a number of studies of the transition from laminar flow to dynamic complexity with increasing $R e$, but for the most part these have been computationally restricted to axisymmetric flows (e.g. Lopez \& Perry 1992; Sorensen \& Christensen 1995; Lopez, Marques \& Sanchez 2001). More recently, three-dimensional nonlinear studies have also been conducted at aspect ratios larger than is considered here (Blackburn \& Lopez 2000, 2002; Marques \& Lopez 2001; Serre \& Bontoux 2002). The choice of aspect ratio in this study is partially guided by the linear stability analysis of this axisymmetric basic state to general three-dimensional disturbances conducted by Gelfgat, Bar-Yoseph \& Solan (2001), who showed that at least for $\Gamma \in[1,3.5]$, all primary instabilities of the axisymmetric basic state are Hopf bifurcations at which stable time-periodic solutions are created. Their stability analysis showed that for $\Gamma$ about 2.7, the basic state becomes unstable to several distinct modes with various azimuthal wavenumbers at critical Reynolds numbers which are quite close together, and that there are a number of closely clustered codimension-two points (points in $\Gamma-R e$ space) at which pairs of such modes simultaneously bifurcate. In the neighbourhood of such points in parameter space, mixed-modes and possibly more 
complex structures also bifurcate (Guckenheimer \& Holmes 1997; Kuznetsov 1998). Even though at onset these are typically unstable (they bifurcate supercritically from a basic state which is already unstable), they compete, interact and some become stable; see Lopez et al. (2001) for an example restricted to the axisymmetric subspace and the corresponding experimental results in Stevens, Lopez \& Cantwell (1999) and three-dimensional computations in Blackburn \& Lopez (2002). In order to avoid as far as possible the complications due to the multiple mode interactions encountered for $\Gamma \geqslant 2.5$, in this present study we fix $\Gamma=1.72$, which from the results of Gelfgat et al. (2001), the mode interaction is from a double Hopf bifurcation from the stable basic state and other mode interactions are further away in $\Gamma-R e$ space. Of course, such complications are inevitable, and we shall show that the mode interaction from the double Hopf at $\Gamma \approx 1.6$ (Marques, Lopez \& Shen 2002) plays a major role in the transition to complex dynamics as $R e$ is increased for $\Gamma=1.72$.

The only symmetry in our problem is the invariance to rotations about the axis. The transition process involves rotating and modulated rotating waves as this symmetry is broken. Such states have been observed experimentally in many diverse problems (e.g. Coles 1965; Hide \& Titman 1967; Gorman \& Swinney 1982). Dynamical systems theory, and in particular the theory on the bifurcations from rotating waves in symmetric systems (e.g. Rand 1982; Golubitsky, LeBlanc \& Melbourne 2000) provides a valuable framework in which to describe and understand the transition process. The theory has been very useful in describing the transition processes in, for example, cellular flames (Bayliss, Matkowsky \& Riecke 1994; Gorman et al. 1994, 1996) and rotating flows (Gorman, Swinney \& Rand 1982). We use this theoretical framework to help clarify the various stages in the transition process from steady axisymmetric flow to flow in which all the symmetries have been broken and the flow has three distinct time scales that differ from one another by about one order of magnitude. The slowest time scale is associated with the underlying precession of the non-axisymmertic flow, the fastest time scale is a modulation of the precession and the third intermediate time scale appears to be due to separation of the cylinder wall boundary layer. At the highest Reynolds numbers considered, we find bursting dynamics at the intermediate time scale with the fast modulations dominating in between the bursts.

\section{Navier-Stokes equations and the numerical scheme}

We consider an incompressible flow confined in a cylinder of radius $R$ and height $H$, driven by the constant rotation of the bottom endwall at $\Omega \mathrm{rad} \mathrm{s}^{-1}$. The system is nondimensionalized using $R$ as the length scale and $1 / \Omega$ as the time scale. The equations governing the flow are the Navier-Stokes equations together with initial and boundary conditions. In cylindrical coordinates, $(r, \theta, z)$, we denote the non-dimensional velocity vector and pressure by $\boldsymbol{u}=(u, v, w)$ and $p$, respectively. The system is governed by two non-dimensional parameters, one geometric and one dynamic:

$$
\begin{array}{ll}
\text { aspect ratio: } & \Gamma=H / R, \\
\text { Reynolds number: } & R e=\Omega R^{2} / \nu,
\end{array}
$$

where $v$ is the kinematic viscosity of the fluid. Note that the viscous time, $R^{2} / v$, non-dimensionalized by $1 / \Omega$, is $R e$.

The governing equations are the (non-dimensional) Navier-Stokes equations

$$
\partial \boldsymbol{u} / \partial t+(\boldsymbol{u} \cdot \nabla) \boldsymbol{u}=-\nabla p+\nabla^{2} \boldsymbol{u} / \operatorname{Re}, \quad \nabla \cdot \boldsymbol{u}=0,
$$


subject to no-slip boundary conditions. Specifically, $u=v=w=0$ on all stationary boundaries, i.e. at the outer cylinder, $r=1$, and the top endwall $z=\Gamma$. On the rotating bottom endwall at $z=0, u=w=0$ and $v=r$.

To solve (2.1), a stiffly stable semi-implicit second-order projection scheme is used, where the linear terms are treated implicitly while the nonlinear terms are explicit (see Lopez \& Shen 1998; Lopez, Marques \& Shen 2002b, for more details). For the space variables, we use a Legendre-Fourier approximation. More precisely, the azimuthal direction is discretized using a Fourier expansion with $N_{\theta}+1$ modes corresponding to azimuthal wavenumbers $m=0,1,2, \ldots, N_{\theta}$, while the axial and radial directions are discretized with a Legendre expansion. The code has already been used to study flows in the present geometry (Marques \& Lopez 2001; Marques et al. 2002; Lopez et al. 2002a; Lopez \& Marques 2004), and these papers give further details on accuracy and resolution. The results presented here have 48 Legendre modes in the radial and axial directions, and $N_{\theta}=15$. The time step used is $\delta t=5 \times 10^{-3}$.

\section{Basic flow state and its Hopf bifurcations}

The rotation of the endwall introduces angular momentum into the flow. For any non-zero rotation of the endwall (i.e. $R e \neq 0$ ), the boundary-layer flow has a radial flow component which advects angular momentum radially outward; the presence of the stationary cylinder turns this flow into the interior and a meridional overturning swirling flow is established which redistributes the angular momentum introduced at the rotating endwall throughout the interior of the cylinder (see figure 1). For sufficiently large Reynolds number $(R e \sim 1000)$, the meridional flow brings enough angular momentum in toward the axis so as to establish a central vortex flow. As $R e$ is increased further, a greater amount of angular momentum is advected toward the axis, resulting in a vortex flow on the axis with a centrifugally unstable distribution of angular momentum. The flow redistributes this angular momentum by establishing a large radial flow out from the axis. By $R e=1500$, this radial outflow is sufficiently strong to stagnate the axial flow and a recirculation zone (vortex breakdown bubble) is formed on the axis. With increasing $R e$, this process occurs over a shorter axial distance down from the top stationary endwall, with the effect of squeezing the nearspherical bubble at lower $R e$ into a cup-shaped bubble $(R e \sim 2000)$, and ultimately squeezing it out altogether. For these higher $R e \sim 2500$, there is flow down the entire length of the axis, but there is still a very strong radial outflow advecting angular momentum away from the axis, and the axial flow near the axis downstream of this is very slow. The flow shown in figure 1 at $R e=2650$ is of the steady axisymmetric basic state at a Reynolds number slightly below that at which the basic state becomes unstable, for $\Gamma=1.72$. Throughout this study, we shall keep $\Gamma=1.72$ and study the instabilities of the flow as $R e$ is increased. Before doing so, it is instructive to review how the symmetry of the problem affects its instabilities and bifurcations.

The system is $S O(2)$ equivariant. The action of $S O(2)$ on the velocity field is that of a rotation $R_{\alpha}$ through angle $\alpha \in(0,2 \pi]$ :

$$
R_{\alpha} \boldsymbol{u}(\boldsymbol{x})=R_{\alpha}(u, v, w)(r, \theta, z, t)=(u, v, w)(r, \theta+\alpha, z, t) .
$$

For an axisymmetric flow field with velocity $\boldsymbol{u}_{a}, R_{\alpha} \boldsymbol{u}_{a}=\boldsymbol{u}_{a}$. The generic codimensionone local bifurcations that such a flow can suffer are the saddle-node bifurcation and the Hopf bifurcation. Saddle-node bifurcations of axisymmetric flows are only possible if the bifurcation preserves the symmetry (Crawford \& Knobloch 1991; Knobloch 1994, 1996; Iooss \& Adelmeyer 1998). Saddle-node bifurcations of the basic state 

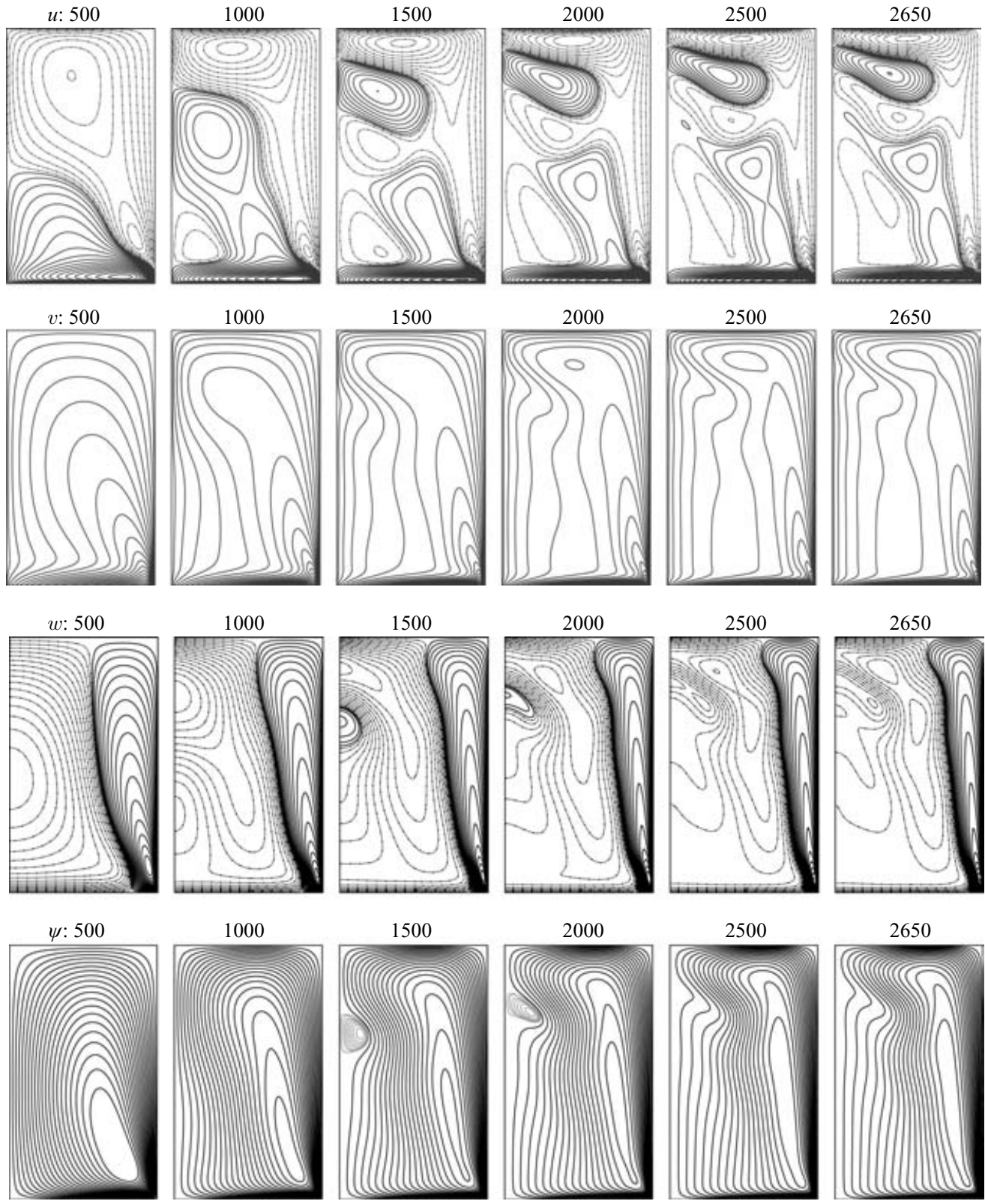

FIGURE 1. Contours of velocity components $u, v$ and $w$, and streamfunction $\psi$ for the axisymmetric steady-state solution at various $R e$ as indicated; solid (broken) contours are positive (negative) with values $\pm 0.16(i / 20)^{2}, i \in[1,20]$, for $u$ and $w$, and $(i / 20)^{2}$ for $v$; for $\psi$, the black contours, in the interval $[-0.01,0)$, indicate counterclockwise circulation and the grey contours in the interval $\left(0,2 \times 10^{-4}\right]$, indicate clockwise recirculation zones. The left-hand boundary is the axis and the bottom is the rotating endwall.

have not been observed experimentally (either in the laboratory or in computations). The linear stability analysis of the axisymmetric basic state to general threedimensional disturbances (Gelfgat et al. 2001) showed that at least for $\Gamma \in[1,3.5]$, all primary instabilities of the axisymmetric basic state are Hopf bifurcations at 
which stable time-periodic solutions are created. For $\Gamma \in(1.63,2.76)$, Gelfgat et al. (2001) showed that the periodic solutions are axisymmetric limit cycles, i.e. the Hopf bifurcation preserves the $S O(2)$ symmetry, and in an earlier study Gelfgat et al. (1996) showed that this Hopf bifurcation is supercritical, while for $\Gamma<1.63$ and $\Gamma>2.76$ the Hopf bifurcation breaks the $S O(2)$ symmetry and a stable rotating wave with azimuthal wavenumber $m \neq 0$ is created.

Rotating waves are periodic solutions which are special in the sense that the timedependence is a drift in the direction of the $S O(2)$ symmetry, i.e. a precession, and are better described as relative equilibria. The velocity field of a rotating wave $\boldsymbol{u}_{\mathrm{rw}}$ in a system with $S O(2)$ symmetry has the following characteristics:

$$
\left.\begin{array}{l}
\boldsymbol{u}_{\mathrm{rw}}(r, \theta, z, t)=\boldsymbol{u}_{\mathrm{rw}}(r, \theta, z, t+T), \\
\boldsymbol{u}_{\mathrm{rw}}(r, \theta, z, t)=\boldsymbol{u}_{\mathrm{rw}}(r, \theta+2 \pi / m, z, t), \\
\boldsymbol{u}_{\mathrm{rw}}(r, \theta, z, t)=R_{-\phi} \boldsymbol{u}_{\mathrm{rw}}(r, \theta, z, t+T \phi / 2 \pi) .
\end{array}\right\}
$$

The Hopf bifurcation from the basic state leading to the rotating wave breaks the continuous $S O(2)$ symmetry, and the rotating wave possesses the discrete cyclic symmetry $Z_{m}=\left\{R_{2 \pi}, R_{2 \pi / 2}, \ldots, R_{2 \pi / m}\right\}$. In a frame-of-reference that rotates with period $T$, the rotating wave is stationary, hence the terminology 'relative equilibrium'.

Near a relative equilibrium the drift dynamics associated with the precession is trivial and decouples from the dynamics orthogonal to the relative equilibrium. As a result, the bifurcations from relative equilibria can be analysed in two steps, describing first the bifurcations associated to the orthogonal dynamics, and then adding the corresponding drift along the rotating wave (Krupa 1990).

To characterize the flow states computed, we use both a local measure and a global measure. The need for both a local and global measure will become apparent as we analyse the results in subsequent sections. The local measure is the value of a component of the velocity at a point (owing to the flow incompressibility, the choice of velocity component and the point at which it is measured is quite arbitrary, as long as the point is not on the axis or the solid walls). We shall monitor $U$, the radial component of the velocity at the point $(r, \theta, z)=(0.5,0,0.5 \Gamma)$. For global measures, we use modal energies:

$$
E_{m}=\frac{1}{2} \int_{z=0}^{z=\Gamma} \int_{r=0}^{r=1} \boldsymbol{u}_{m} \cdot \overline{\boldsymbol{u}}_{m} r \mathrm{~d} r \mathrm{~d} z,
$$

where $\boldsymbol{u}_{m}$ is the $m$ th Fourier mode of the velocity field. For an axisymmetric solution, only $E_{0}$ is non-zero, and for a rotating wave with $Z_{m}$ spatial symmetry, only $E_{k m}$ are non-zero $(k=0,1,2, \ldots)$.

\subsection{Axisymmetric limit cycles}

For $\Gamma$ about 1.72 , the basic state loses stability via symmetry-preserving Hopf bifurcations as $R e$ is increased beyond about 2660. The linear stability analysis of Gelfgat et al. (2001) predicts that the frequency of the bifurcating limit cycle changes discontinuously with $\Gamma$ in this region of parameter space, indicating a codimensiontwo double Hopf bifurcation at about $\Gamma=1.725$ and $R e=2655$. For $\Gamma<1.725$, the limit cycle LC1 is the first to bifurcate from the basic state as $R e$ is increased and for $\Gamma>1.725$, LC2 is the primary mode. Near onset, both limit cycles show periodic pulsations of the vortex breakdown region (the region where flow on the central vortex near the top stationary wall makes a sudden radial expansion, as described for the basic state; see figure 1). For LC1, these pulsations are of large amplitude and 
(a)
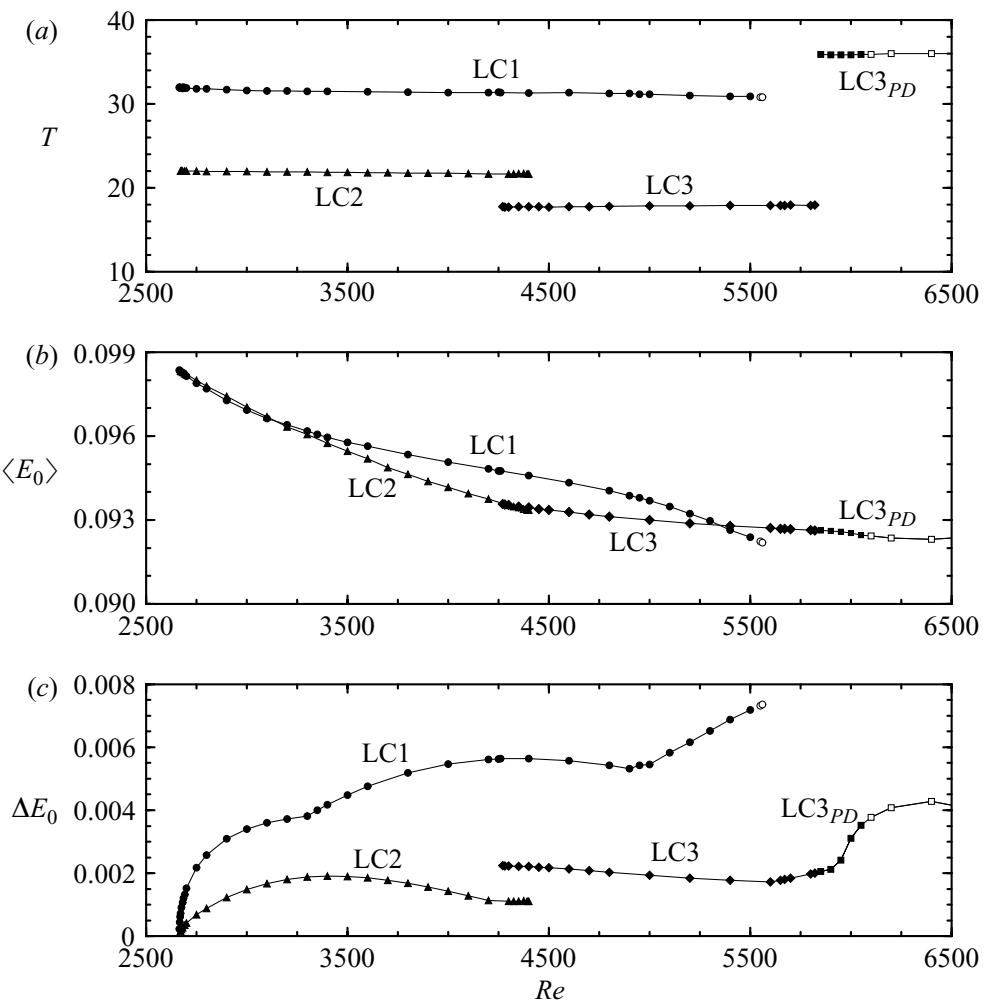

FiguRE 2. Variation with $R e$ of $(a)$ the periods $T,(b)\left\langle E_{0}\right\rangle$ and $(c) \Delta E_{0}$, of the axisymmetric limit cycle states LC1, LC2 and LC3, as well the period-doubled LC3 $P D$. Solid symbols are computed solutions which are stable to three-dimensional perturbations and open symbols are computed solutions for which modes with $m \neq 0$ are growing.

short period compared with the pulsations of LC2. Their character is of the same pulsation type as the primary Hopf mode observed at $\Gamma=2.5$ (Lopez \& Perry 1992; Stevens et al. 1999; Lopez et al. 2001). The frequencies of the observed limit cycles are in very good agreement with the Hopf frequencies predicted by the linear stability analysis. Figure 2 shows how the periods $T,\left\langle E_{0}\right\rangle$ (the mean $E_{0}$ over one period), and $\Delta E_{0}$ (the amplitude of the oscillation in $E_{0}$ ) vary with $R e$ for the axisymmetric limit cycles. As $R e$ is increased, the amplitude of the oscillations increases and the nature of the pulsations changes so that a recirculation zone propagates down the axis every period following the expansion phase of the pulsation for both LC1 and LC2 (see figures 3 and 4 for contours of the axial velocity for LC1 and LC2 at $R e=4000$ ). This wave travelling down the axis is reminiscent of the second Hopf mode observed both experimentally and numerically at $\Gamma=2.5$ (Lopez \& Perry 1992; Stevens et al. 1999; Lopez et al. 2001; Blackburn \& Lopez 2002).

By solving the three-dimensional Navier-Stokes equations with initial conditions that include finite perturbations in the $m=1$ Fourier mode, and monitoring $E_{m}$ for $m \neq 0$, we have found that both $\mathrm{LC} 1$ and LC2 remain stable to quite large $R e$. LC1 is stable for $R e \leqslant 5500$; for slightly larger $R e$, the non-axisymmetric components grow slowly. The computations indicate that LC1 loses stability via a symmetrybreaking Neimark-Sacker bifurcation (Hopf bifurcation of limit cycles), and that the resulting quasi-periodic state is itself unstable, indicating that the bifurcation is 

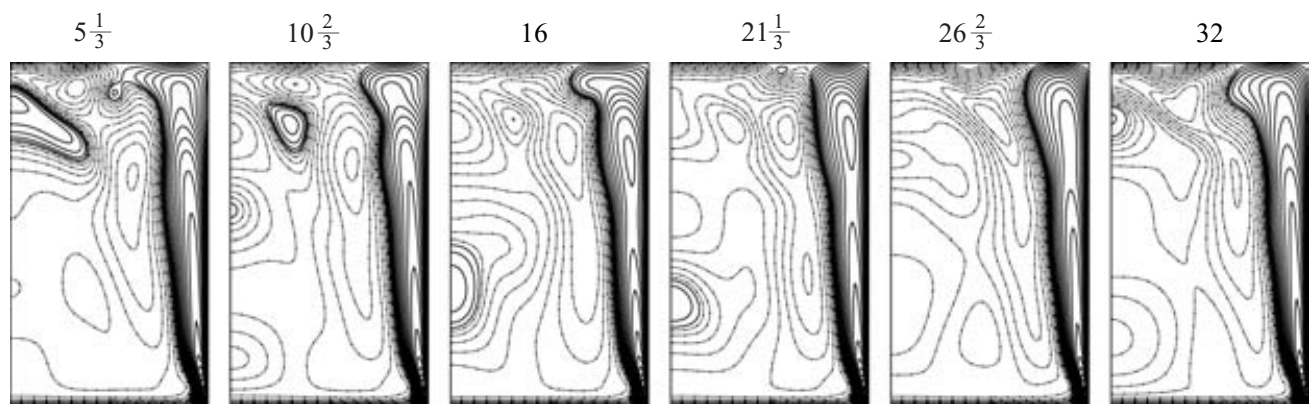

FIgURE 3. Contours of $w$ at different times $t$ over one period $T \approx 32$, as indicated, for LC1 at $R e=4000$; solid (broken) contours are positive (negative) with values $\pm 0.16(i / 20)^{2}, i \in[1,20]$.
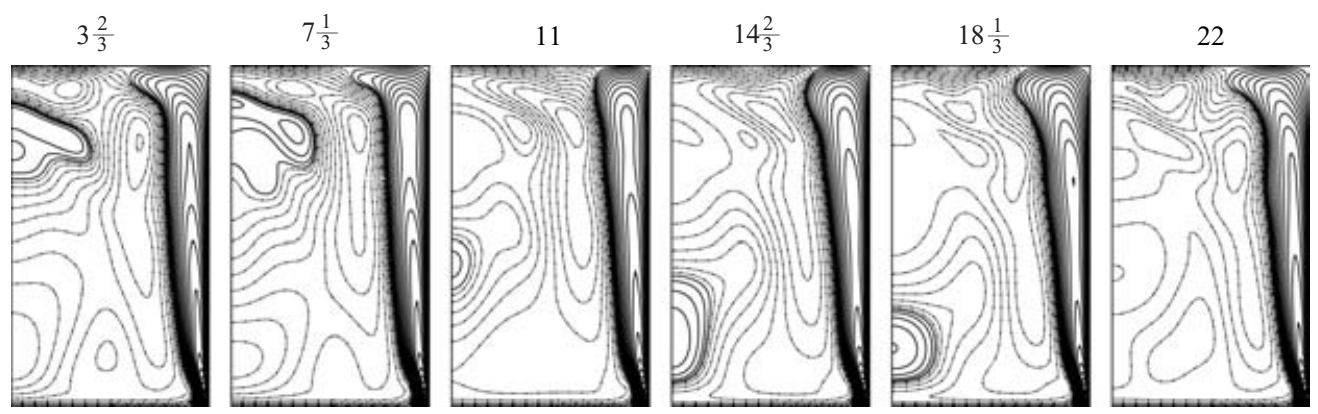

FIGURE 4. Contours of $w$ at different times $t$ over one period $T \approx 22$, as indicated, for LC2 at $R e=4000$; solid (broken) contours are positive (negative) with values $\pm 0.16(i / 20)^{2}, i \in[1,20]$.

subcritical. After several viscous times, the flow evolves to a non-axisymmetric state which we describe later. We could continue the unstable LC1 solution branch to higher $R e$ by restricting the computations to the axisymmetric subspace (by solving the axisymmetric Navier-Stokes equations), and study the complex dynamics the axisymmetric flow experiences with increasing $R e$, such as was done by Sorensen \& Christensen (1995) for $\Gamma=2.0$.

The LC2 branch loses stability at lower $R e \approx 4400$. This bifurcation is a saddle-node of limit cycles. Above $R e=4400$, there is a jump to a different branch of axisymmetric limit cycles, LC3, which can be followed to both larger and smaller $R e$. Following this branch to smaller $R e$, it also suffers a saddle-node of limit cycles bifurcation at about $R e=4265$ and there is a jump back to the LC2 branch, giving the typical hysteresis loop associated with saddle-node bifurcations. The characteristics of the LC3 branch are similar to those of LC1 and LC2 at comparable $\operatorname{Re}$ (see figures 3 and 4), consisting of a recirculation zone that forms on the axis just downstream of the breakdown region and travels at a fairly constant speed down the axis; as it reaches the rotating endwall, a new recirculation zone forms and the whole process repeats. Figure 5 shows contours of the axial velocity over one period, illustrating this process at $R e=5800$.

At $R e \approx 5840$, LC3 undergoes a period-doubling bifurcation to $\mathrm{LC}_{P D}$. The amplitudes of the limit cycles are continuous across this bifurcation, but the period suddenly doubles. The flow field characteristics of the period-doubled solution are very similar to those of LC3 (compare figures 5 and 6). Figure 7 shows time-series of $E_{0}$ for LC3 at $R e=5800$ (solid curve) and for $\mathrm{LC}_{P D}$ at $R e=6000$ (dashed curve). At 

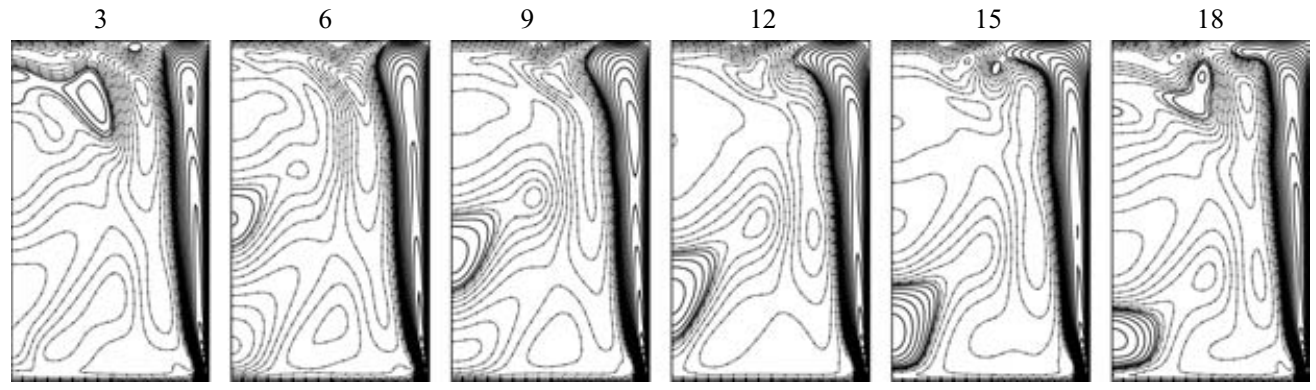

Figure 5. Contours of $w$ at different times $t$ over one period $T \approx 17.91$, as indicated, for LC3 at $R e=5800$; solid (broken) contours are positive (negative) with values $\pm 0.16(i / 20)^{2}$, $i \in[1,20]$.

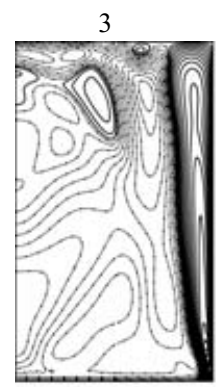

21
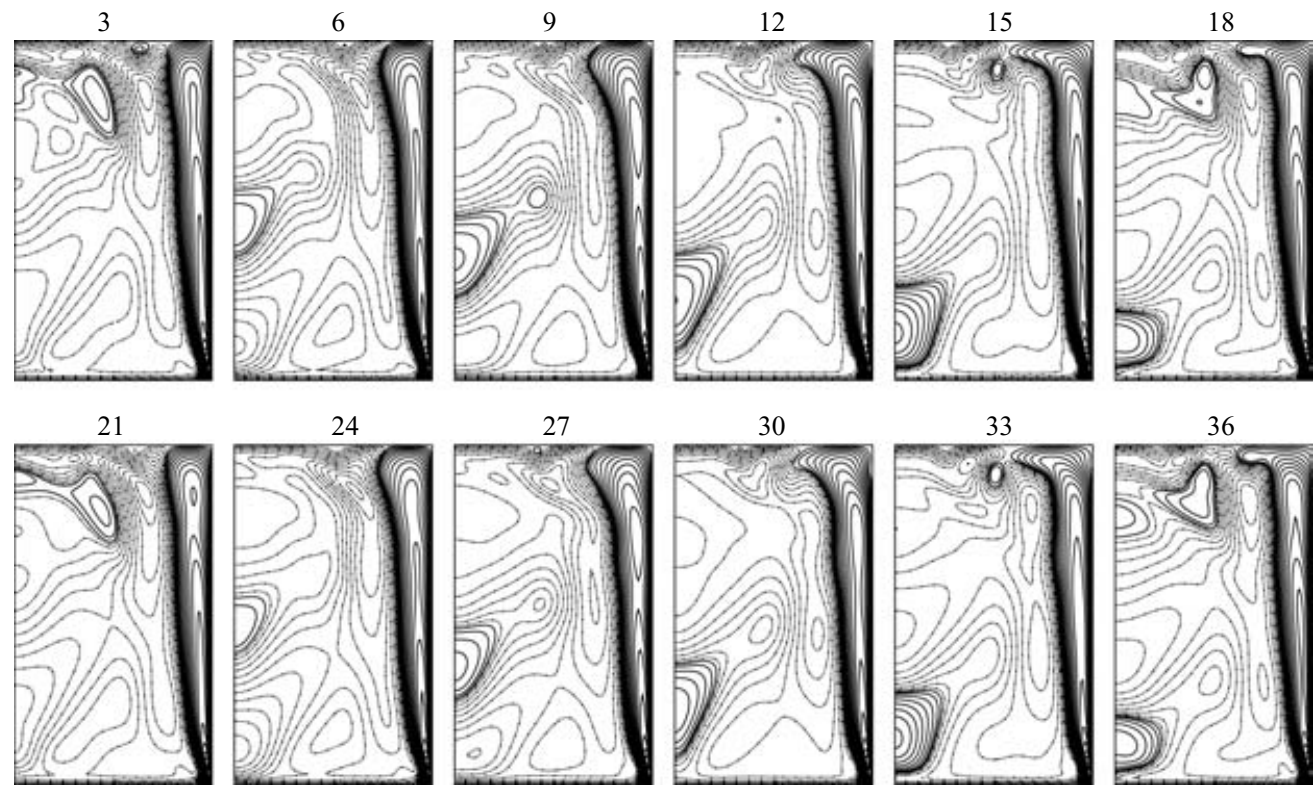

FIgURE 6. Contours of $w$ at different times $t$ over one period $T \approx 35.86$, as indicated, for $\mathrm{LC} 3_{P D}$ at $R e=6000$; solid (broken) contours are positive (negative) with values $\pm 0.16(i / 20)^{2}$, $i \in[1,20]$.

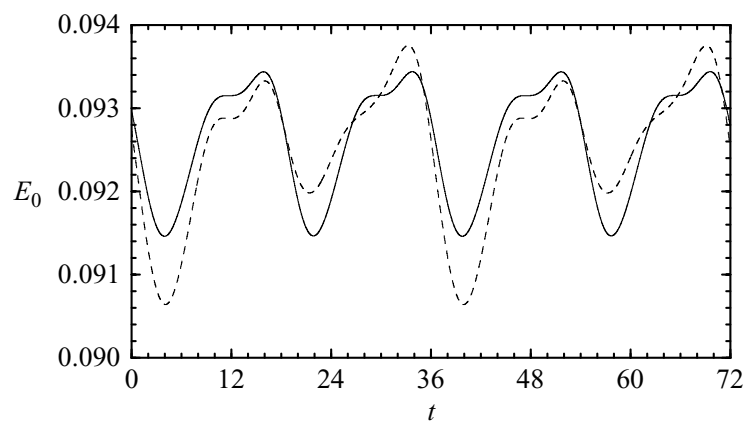

FIGURE 7. Temporal variation of $E_{0}$ for LC3 at $R e=5800$ (solid curve, with period $T \approx 17.91$ ) and $\mathrm{LC} 3_{P D}$ at $R e=6000$ (dashed curve, with period $T \approx 35.86$ ). 
about $R e=6050, \mathrm{LC} 3_{P D}$ becomes unstable to non-axisymmetric perturbations. This appears to be via a symmetry-breaking Neimark-Sacker bifurcation as we are able to continue the unstable $\mathrm{LC} 3_{P D}$ branch to higher $R e$ by restriction to the axisymmetric subspace.

\subsection{Rotating waves}

When LC1 and $\mathrm{LC} 3_{P D}$ become unstable to non-axisymmetric perturbations, the flows evolve to three-dimensional states which we shall describe later. By continuing these states to lower $R e$, we have found a branch of rotating waves with azimuthal wavenumber $m=2$, RW2. This branch of RW2 loses stability for $R e<3425$; for smaller $R e$, the flow evolves to the LC2 state. The loss of stability of RW2 as $R e$ is reduced is via a Hopf bifurcation from the rotating wave; the bifurcation is a Hopf bifurcation from a (relative) equilibrium rather than a Neimark-Sacker (Hopf bifurcation for maps) bifurcation because the time-periodic state RW2 is a relative equilibrium and so the time-periodicity is simply a drift in the direction of the $S O(2)$ symmetry, i.e. a uniform precession, which is decoupled from the dynamics orthogonal to the group orbit (Rand 1982; Renardy 1982; Krupa 1990; Golubitsky et al. 2000). The quasi-periodic state that emerges at the bifurcation of RW2 is unstable, and we have no details about it. The unstable RW2 continues to exist to lower $R e$. From the linear stability analysis of Gelfgat et al. (2001), for $\Gamma=1.72$, RW2 bifurcates from the basic state (which is already unstable to both LC1 and LC2) at about $R e=2800$, and the Hopf frequency is a very small negative number (see their figures 1 and 2) indicating that at onset, RW2 has a very slow retrograde precession with respect to the sense of rotation of the endwall. At $R e \approx 3425$, the stable RW2 is precessing very slowly prograde with the endwall. Presumably, for Re between 2800 and 3400, the sense of precession of the unstable RW2 changes.

Figure 8 shows the variation with $R e$ of the mean modal energies $\left\langle E_{0}\right\rangle,\left\langle E_{1}\right\rangle$ and $\left\langle E_{2}\right\rangle$ and the precession and modulation periods of RW2 and various modulated rotating waves that result from subsequent bifurcations (these are discuss in detail later). The precession period $T$ corresponds to the time it takes for the RW2 structure to precess through angle $2 \pi$. Since RW2 has spatial symmetry $Z_{2}=\left\{\mathbf{1}, R_{\pi}\right\}$, an observable such as a velocity component at a point has periodicity $T / 2$. Furthermore, the time series of such a velocity component is not harmonic. This does not mean that RW2 has a non-uniform precession. The time series would be harmonic if RW2 were completely described by just the $m=0$ and $m=2$ Fourier modes, but this is the case only at the Hopf bifurcation point where RW2 is spawned. The non-harmonicity is due to the nonlinear spatial structure of RW2, as can be readily seen in figure 9. This figure, which shows contours of the radial velocity at various heights for $R e=4500$, also illustrates the $Z_{2}$ symmetry of RW2. These contours rotate rigidly in space in the counterclockwise direction (prograde with the rotating endwall) at a constant rate $\dot{\theta}=2 \pi / T$. Figure 10 shows contours of the axial velocity at various angles $\theta \in[0, \pi)$; the contours are repeated for $\theta \in[\pi, 2 \pi)$ because of the $Z_{2}$ symmetry. The contours of $u$ at $z=0.8 \Gamma$ in figure 9 illustrate the coupling between the strong radial outflow associated with the vortex breakdown and the boundary-layer flow near the cylinder wall. The contours in figure 10 also illustrate the activity in the corner region $(r=1, z=\Gamma)$, where the sidewall boundary layer turns to form the stationary endwall layer; there is a pair (owing to the $Z_{2}$ symmetry) of small spiral separation zones on the stationary top endwall (most clearly seen in the contours at $\theta=3 \pi / 6)$. 

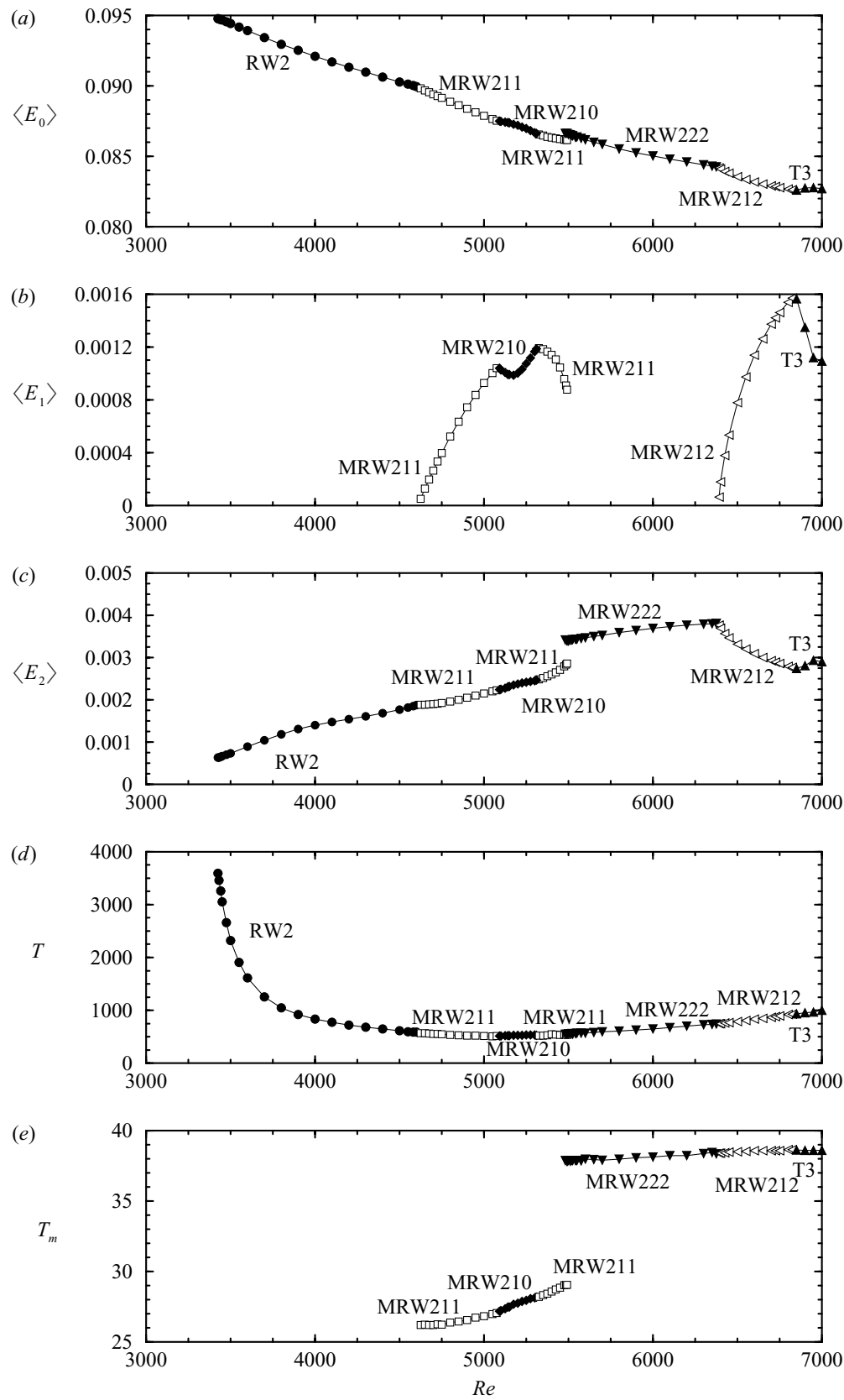

FiguRE 8. Variation with $R e$ of the mean modal energies $\left\langle E_{0}\right\rangle,\left\langle E_{1}\right\rangle$ and $\left\langle E_{2}\right\rangle$ and the precession and modulation periods, $T$ and $T_{m}$, of RW2 and various modulated rotating waves (as labelled) that result from subsequent bifurcations.

\subsection{Relative periodic orbits and modulated rotating waves}

As RW2 is continued to higher $R e$, it becomes unstable at about $R e=4610$. An $m=1$ Fourier mode grows and the solution is a quasi-periodic modulated rotating wave. Figure 8 shows the variation with $R e$ of $\left\langle E_{i}\right\rangle, i=0,1,2$ for the modulated rotating wave, showing the growth of $\left\langle E_{1}\right\rangle$ from zero at the instability of RW2; 

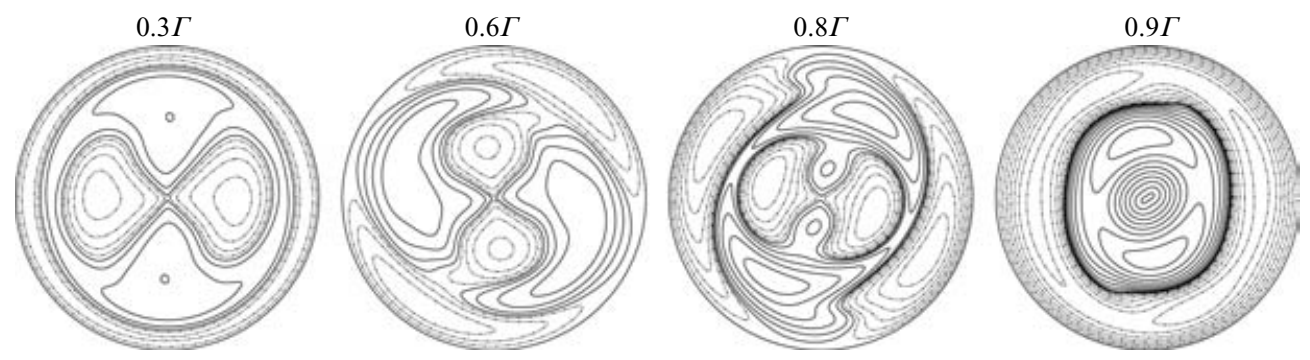

Figure 9. Contours of $u$ at different heights $z$, as indicated, for RW2 at $R e=4500$; solid (broken) contours are positive (negative) with values $\pm 0.16(i / 20)^{2}, i \in[1,20]$. The sense of precession is counterclockwise (the same sense as the rotation of the bottom endwall).
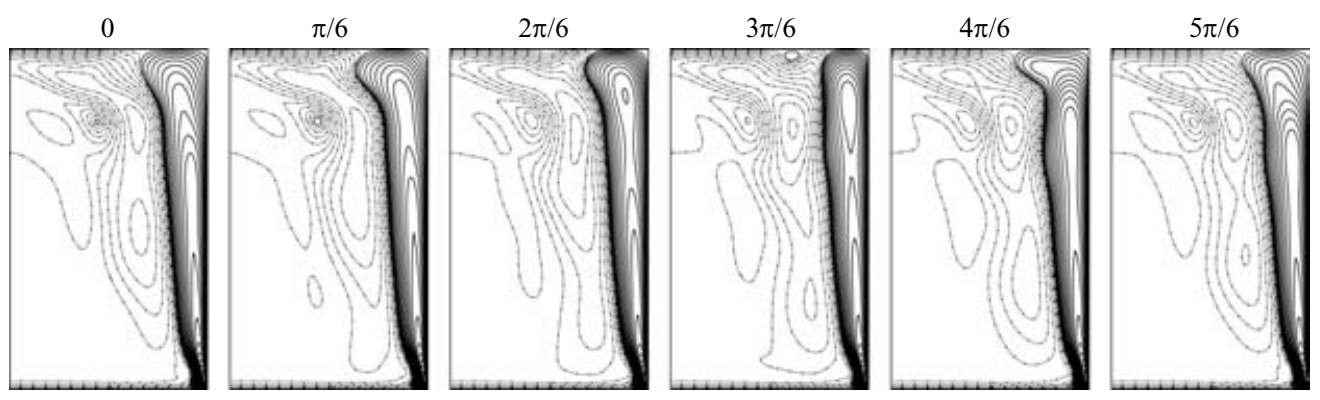

FIGURE 10. Contours of $w$ at different azimuthal angles, as indicated, at one instant in time, for RW2 at $R e=4500$; solid (broken) contours are positive (negative) with values $\pm 0.16(i / 20)^{2}$, $i \in[1,20]$.

the variations of $\left\langle E_{0}\right\rangle$ and $\left\langle E_{2}\right\rangle$ for the modulated rotating wave are continuous with those of RW2 across this instability. There is also a destabilization and restabilization of the modulated rotating wave to a different modulated rotating wave for $R e \in(5080,5310)$.

The instability of RW2 could be interpreted as a Neimark-Sacker bifurcation, i.e. a Hopf bifurcation from the time-periodic RW2, but since RW2 is a rotating wave, it is more appropriate to consider this as a Hopf bifurcation from the (relative) equilibrium (Krupa 1990). In this setting, the bifurcating quasi-periodic solution is a modulated rotating wave which is a (relative) periodic orbit in the precessing frame of reference in which the rotating wave is stationary. Rand (1982) has classified the modulated rotating waves that bifurcate via Hopf bifurcations from rotating waves in systems with $S O(2)$ symmetry in terms of the spatial symmetries of the rotating wave and of the modulated rotating wave. Golubitsky et al. (2000) also use the spatio-temporal symmetry of the modulated rotating wave to complete the classification.

As we have already mentioned, RW2 has spatial symmetry $Z_{2}$. The Hopf bifurcation breaks this symmetry, introducing an $m=1$ azimuthal wavenumber and so the bifurcating modulated rotating wave only has trivial spatial symmetry, the flow is periodic in the azimuthal direction. However, from a close examination of the velocity field (see figure $11 a$, showing contours of the axial velocity at $z=0.8 \Gamma$ of the modulated rotating wave at $R e=4800$ over one modulation period), we find that it possesses a non-trivial spatio-temporal symmetry. From the theory of Krupa (1990), the appropriate way to investigate such flows is to examine the dynamics normal to the group orbit (for us this means considering the dynamics in a rotating 


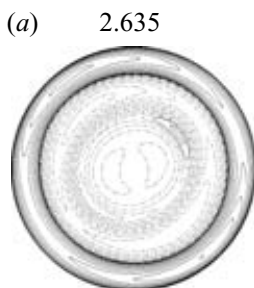

15.810

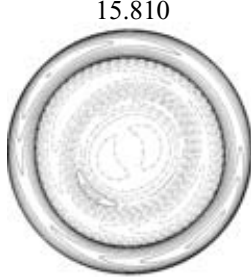

(b) $\quad 2.775$
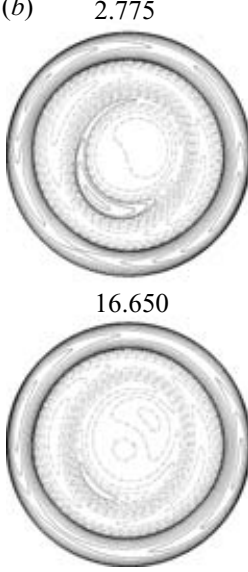

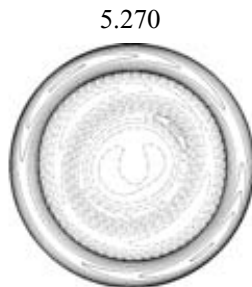

18.445

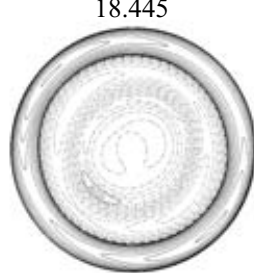

5.550

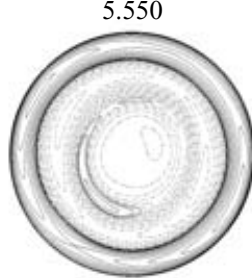

19.425

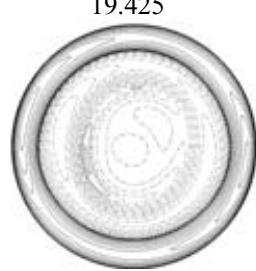

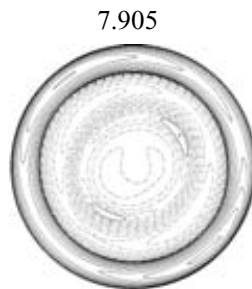

21.080

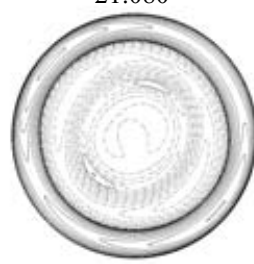

8.325

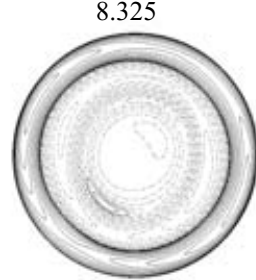

22.200

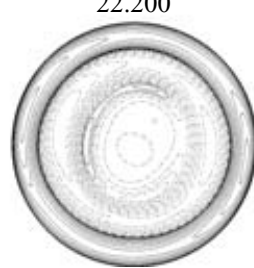

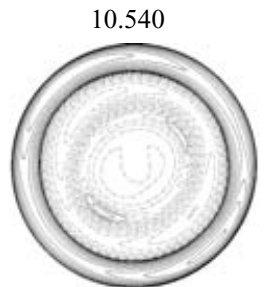

23.715

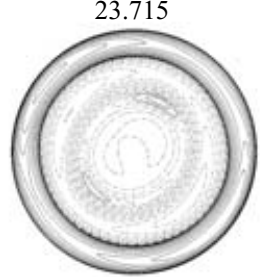

11.100

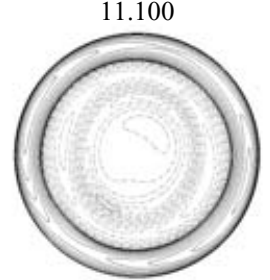

24.975

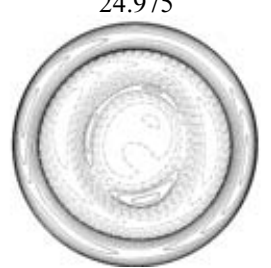

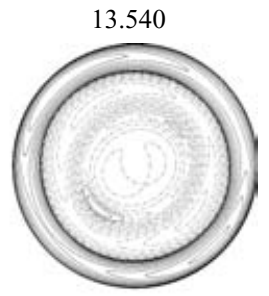

26.350

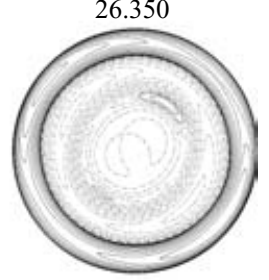

13.875

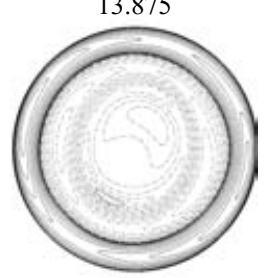

27.750

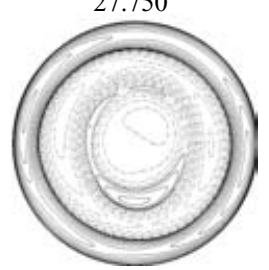

FIGURE 11. Contours of $w$ at $z=0.8 \Gamma$ at times as indicated over one modulation period for (a) MRW211 at $R e=4800$ and (b) MRW210 at $R e=5200$.

frame-of-reference in which the rotating wave is stationary and the modulated rotating wave is periodic). The contours in figure 11 are drawn in the stationary frame, and to eliminate the (counterclockwise) precession, contour plots at time $t$ from a reference plot should be rotated clockwise by angle $2 \pi t / T$, where the precession period $T$ varies with $R e$. The modulation period, $T_{m}$, is about a factor of 20 times smaller than $T$ (figure 8 shows the variation of $T$ and $T_{m}$ with $R e$ ). The velocity in the appropriate rotating frame (i.e. rotating clockwise with period $T(R e)$ ), denoted $\tilde{\boldsymbol{u}}$, has the spatio-temporal symmetry:

$$
R_{\pi} \tilde{\boldsymbol{u}}(r, \theta, z, t)=\tilde{\boldsymbol{u}}(r, \theta+\pi, z, t)=\tilde{\boldsymbol{u}}\left(r, \theta, z, t+T_{m} / 2\right) .
$$

This is analogous to the half-period-flip spatio-temporal symmetry associated with the familiar Kármán vortex street (e.g. Barkley \& Henderson 1996; Marques, Lopez \& Blackburn 2004; Blackburn, Marques \& Lopez 2005), except that instead of a flip ( $Z_{2}$ generated by a spatial reflection), we have $Z_{2}$ generated by a half-period spatial rotation $R_{\pi}$. This spatio-temporal symmetry also gives a $Z_{2}$ group consisting of the identity and the combined operation of a temporal evolution through $T_{m} / 2$ together with $R_{\pi}$, and we denote it $\tilde{Z}_{2}$ to distinguish it from the spatial $Z_{2}$. Since the resulting 

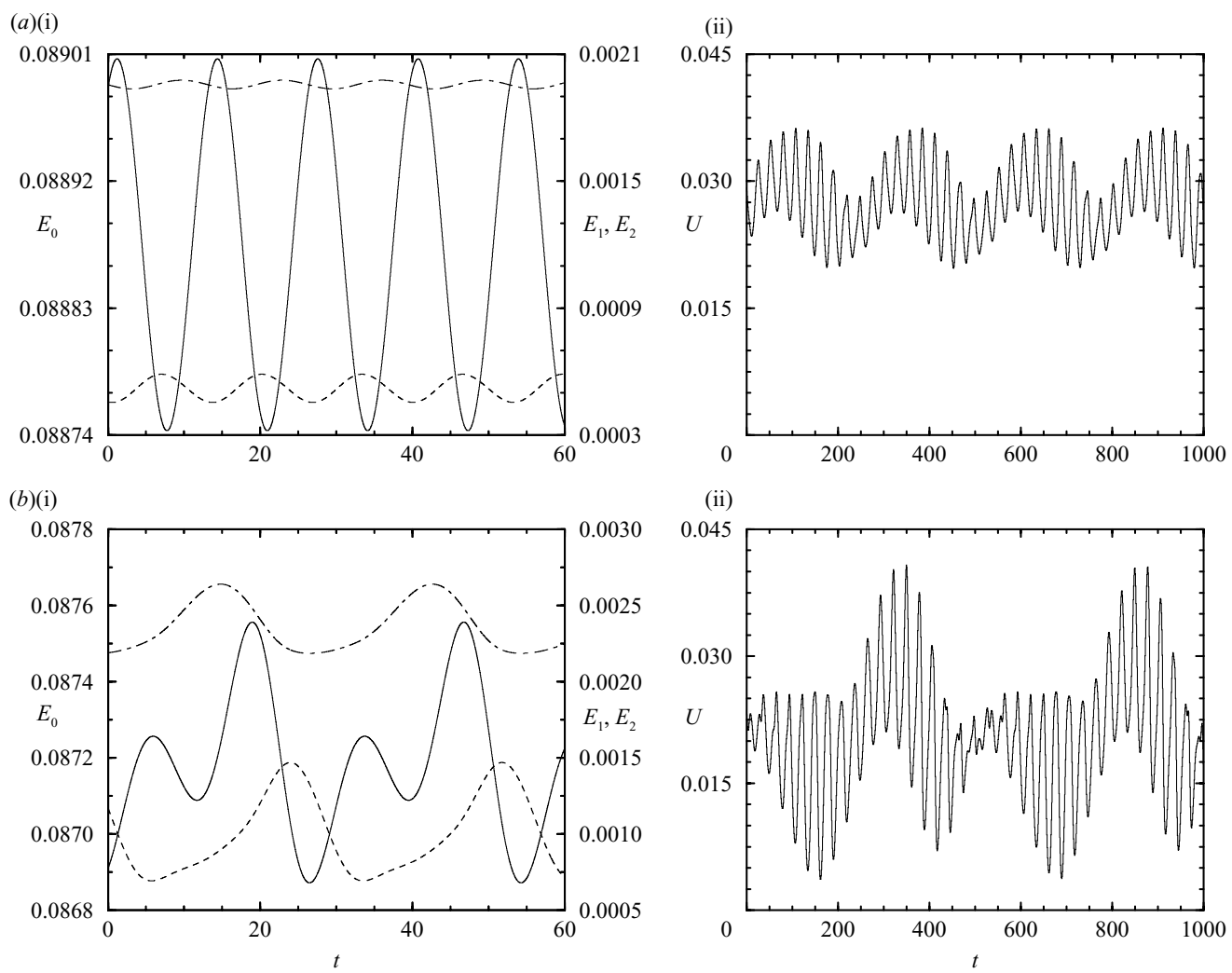

FIgURE 12. Time series of (i) $E_{0}$ (solid line), $E_{1}$ (dashed line), $E_{2}$ (dot-dashed line) and (ii) $U$ for $(a)$ MRW211 at $R e=4800$ and $(b)$ MRW210 at $R e=5200$.

modulated rotating wave has this non-trivial spatio-temporal symmetry, we denote it MRW211. The integer 2 denotes the spatial $Z_{2}$ symmetry of the rotating wave from which it bifurcates, and the first 1 denotes the (trivial) $Z_{1}$ spatial symmetry of MRW211. These are two of the three integers that Golubitsky et al. (2000) used to characterize the modulated rotating waves, and are related to the two integers used by Rand (1982). The second 1 indicates that the modulated rotating wave has $\tilde{Z}_{2}$ spatio-temporal symmetry; Golubitsky et al. (2000) use an integer $\alpha$ which for our MRW211 has the value $\alpha=1$ (they consider more general cases where the spatiotemporal symmetry of the modulated rotating waves can be more complicated than the $\tilde{Z}_{2}$ in MRW211, but later we shall encounter one with $\alpha=2$ ).

Figure 8 shows that for $R e \in(5080,5315)$, MRW211 is unstable to a different type of modulated rotating wave. No new frequencies (either spatial or temporal) are introduced, and the bifurcations of MRW211 at either end of this range in $R e$ are not saddle nodes. The contours of $w$ at $z=0.8 \Gamma$ in figure $11(b)$ indicate that this modulated rotating wave is not invariant to the $\tilde{Z}_{2}$ symmetry (3.4). In the nomenclature of Golubitsky et al. (2000), it has $\alpha=0$, indicating no spatiotemporal symmetry. We shall refer to this state as MRW210 (the 2 due to the spatial $Z_{2}$ symmetry of the underlying rotating wave, the 1 due to the trivial spatial $Z_{1}$ symmetry of MRW210, and the 0 due to its lack of spatio-temporal symmetry).

Figure 12 shows time series of $E_{0}, E_{1}, E_{2}$ and $U$ for MRW211 and MRW210. The $U$ time series are quasi-periodic, with a slow time scale corresponding to the 

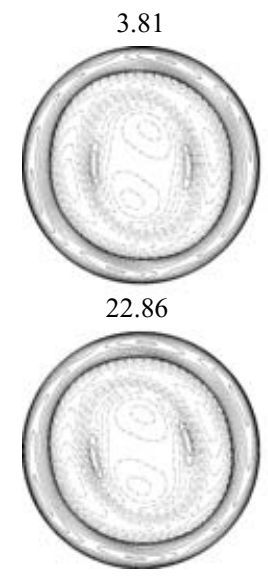

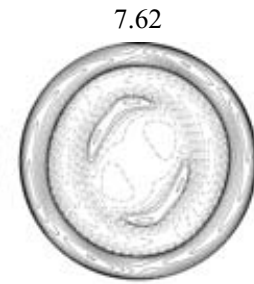

26.67

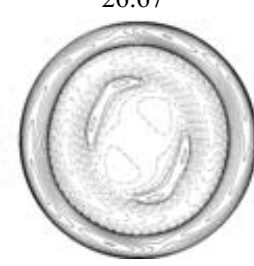

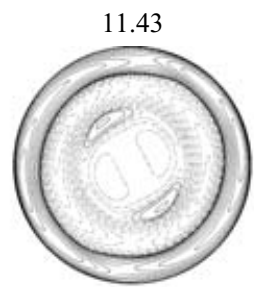

30.48

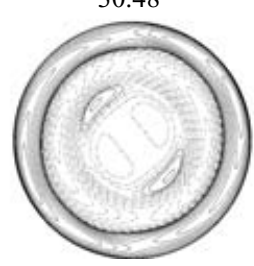

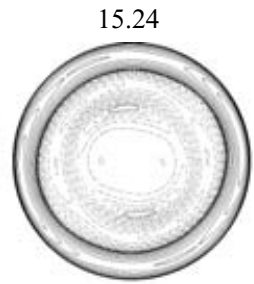

34.29

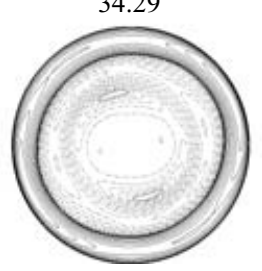

19.05

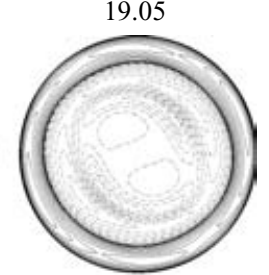

38.10

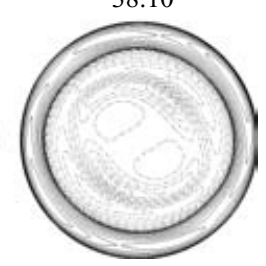

FIGURE 13. Contours of $w$ at $z=0.8 \Gamma$ at times as indicated over approximately one modulation period, $T_{m}=38.12$, for MRW222 at $R e=6000$.

$S O$ (2) drift (the underlying precession of the rotating wave) and a fast time scale corresponding to the modulation. Physically, the modulation is a wobbling of the vortex breakdown region, which is coupled to undulations in the sidewall boundary layer. The $E_{i}$ time series are periodic (since both MRW211 and MRW210 are relative periodic orbits), and their periods are related to the modulation period $T_{m}$. The period $T_{m}$ is the time it takes the modulated rotating wave to repeat in a frame of reference rotating with the precession period. From figure $11(a), T_{m} \approx 26.35$ for MRW211, but the time series of $E_{i}$ in figure $12(a)$ show periodicity of $T_{m} / 2$. This is a consequence of the $\tilde{Z}_{2}$ symmetry; the spatial structure of the velocity at times $T_{m} / 2$ apart are identical, except for a rotation through angle $\pi T_{m} / T$ which does not affect the global measures $E_{i}$ but does affect the local measure $U$. Hence, the fast time scale in the time series of $U$ is $T_{m}$.

For MRW210, $\tilde{Z}_{2}$ symmetry is broken (see figure $11 b$ ) and so the time series of $E_{i}$ (figure $12 b$ ) have periodicity $T_{m}$. The time series of $U$ for MRW210 has fast time scale $T_{m}$ and the slow time scale is the precession period $T$. In contrast, for MRW211, the slow time scale in the time series of $U$ is $T / 2$ as a consequence of the $\tilde{Z}_{2}$ symmetry.

From figure 8, we see that on increasing $R e$ beyond about 5500, MRW211 suffers a saddle-node bifurcation (of relative periodic orbits). For $R e$ slightly beyond the saddle-node bifurcation, the flow has very long transients (several viscous times, which is typical near saddle-node bifurcations), but eventually it evolves to another modulated rotating wave which is distinguished from either MRW211 or MRW210 by its spatial $Z_{2}$ symmetry, such that

$$
\boldsymbol{u}(r, \theta, z, t)=\boldsymbol{u}(r, \theta+\pi, z, t) .
$$

Contour plots of this new state are shown in figure 13. These contours also show that this state has a spatio-temporal symmetry that is different from $\tilde{Z}_{2}$. In a precessing frame-of-reference, it has invariance

$$
\tilde{\boldsymbol{u}}\left(r, \theta, z, t+T_{m} / 2\right)=\tilde{\boldsymbol{u}}(r, \theta, z, t)=\left(R_{\pi}\right)^{2} \tilde{\boldsymbol{u}}(r, \theta, z, t) .
$$

The $\left(R_{\pi}\right)^{2}$ leads to $\alpha=2$ in the nomenclature of Golubitsky et al. (2000) for classifying the spatio-temporal symmetry of modulated rotating waves. The temporal sequence in figure 13 shows that the modulation period is $T_{m}$; following one of the pair of maxima shows that it rotates through angle $-\pi$ in time $T_{m} / 2$ (modulo the small 


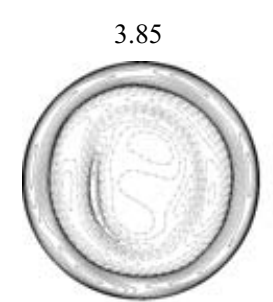

23.10

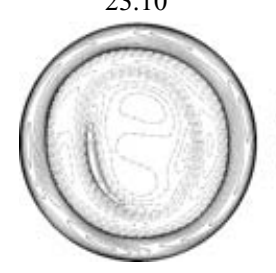

7.70

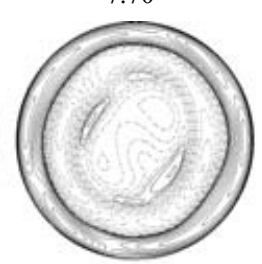

26.95

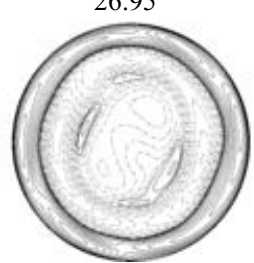

11.55

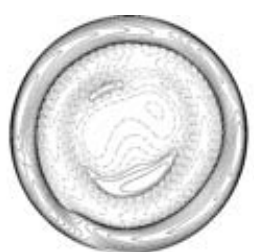

30.80

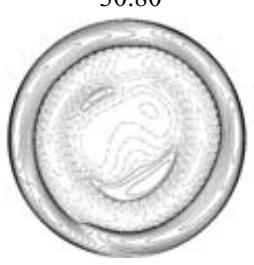

15.40

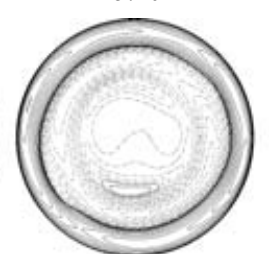

34.65

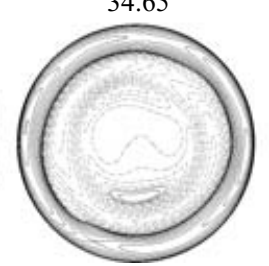

19.25

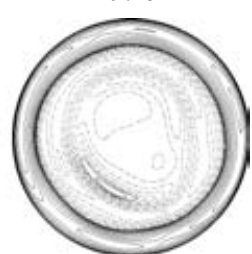

38.50

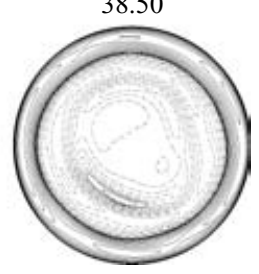

Figure 14. Contours of $w$ at $z=0.8 \Gamma$ at times as indicated over approximately one modulation period, $T_{m}=38.52$, for MRW212 at $R e=6500$.

precession $\left.\pi T_{m} / T\right)$. This modulated rotating wave is related to the underlying RW2 with spatial $Z_{2}$ symmetry, it has spatial $Z_{2}$ symmetry and spatio-temporal symmetry (3.6) with $\alpha=2$, and we denote it MRW222.

Continuing MRW222 to smaller $R e$, we find that it becomes unstable to an $m=1$ Fourier mode at about $R e=5475$, at which point the flow evolves to MRW211. This delineates a hysteresis loop, with the saddle-node bifurcation of MRW211 at about $R e=5495$ at one end and the subcritical Hopf-type bifurcation of MRW222 at about $R e=5475$ at the other end.

Since the stable modulated rotating waves for $R e \in(4610,5475)$ all have $m=1$ Fourier components, it is possible to explore the unstable states from which they bifurcate in this range of $R e$ by restricting the dynamics to an even subspace (computationally, this is done simply by setting all odd Fourier modes identically to zero). This allows us to continue the unstable RW2 state for $R e>4210$ beyond its Hopf bifurcation that spawns MRW211, and for $R e<5475$ we can continue the unstable MRW222. The unstable RW2 and the unstable MRW222 are not simply connected by a single bifurcation in the even subspace. Instead, there is a complicated competition between the unstable MRW222 and another modulated rotating wave which bifurcates from the unstable RW2. We do not present the details as it is probable that the complicated dynamics are an artefact of the restriction to the even subspace. The point, however, is that the branch of stable MRW222 bifurcates (in a non-simple fashion) from the branch of RW2.

Continuing the stable MRW222 to higher $R e$, it suffers a symmetry-breaking bifurcation at about $R e=6350$, spawning another modulated rotating wave where the spatial $Z_{2}$ symmetry is broken owing to the growth of an $m=1$ Fourier mode, but the spatio-temporal symmetry (3.6) is preserved. Figure 14 shows contours of the axial velocity at $z=0.8 \Gamma$ over one modulation period, illustrating the trivial $Z_{1}$ spatial symmetry and the spatio-temporal symmetry (3.6) of this state at $R e=6500$, which we denote MRW212.

Both MRW222 and MRW212 are periodic states in a frame-of-reference precessing at angular rate $\dot{\theta}=2 \pi / T$; owing to the $Z_{2}$ symmetry, the $U$ time series of MRW222 is quasi-periodic with periods $T / 2$ and $T_{m}$, whereas that of MRW212 is quasi-periodic 
(a)(i)
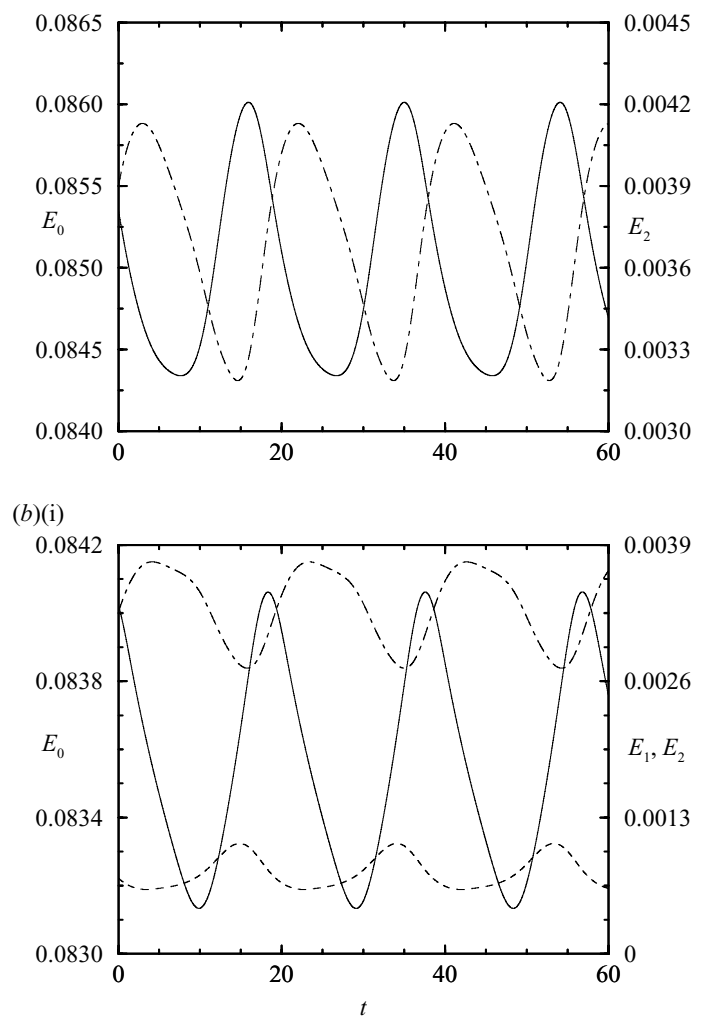

(ii)
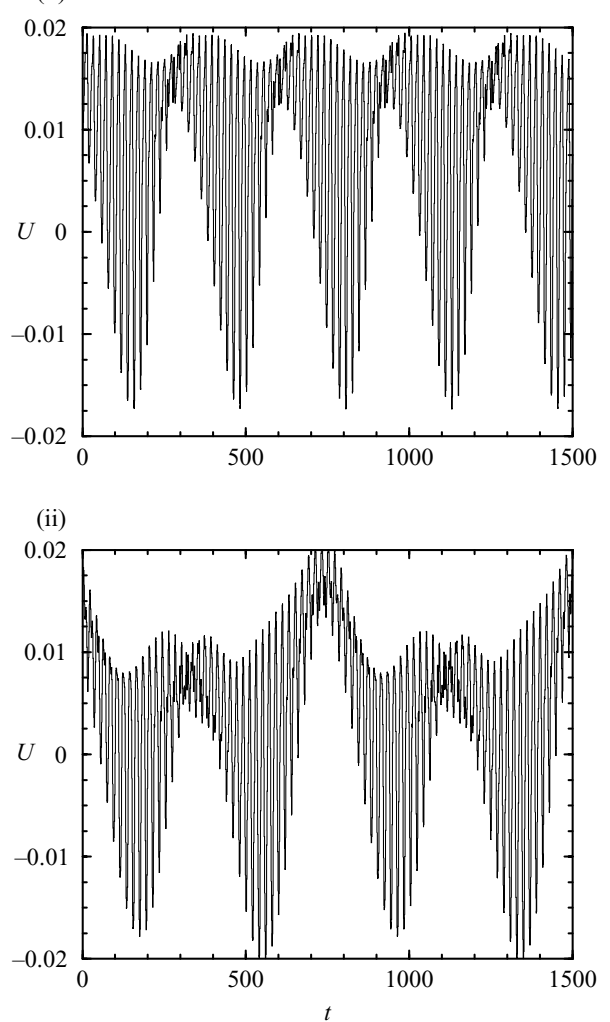

Figure 15. Time series of (i) $E_{0}$ (solid line), $E_{1}$ (dashed line), $E_{2}$ (dot-dashed line) and (ii) $U$ for $(a)$ MRW222 at $R e=6000$ and $(b)$ MRW212 at $R e=6500$.

with periods $T$ and $T_{m}$; and yet, since both are invariant to the spatio-temporal symmetry (3.6), their $E_{i}$ time series are $T_{m} / 2$ periodic (see figure 15).

At about $R e=6830$, MRW212 becomes unstable (see figure 8) at a bifurcation at which a new frequency is introduced; we shall denote this new state T3 as it has three distinct time scales associated with it, as we shall describe presently. Figure 16 shows power spectral densities of $E_{2}$ (the time series used to obtain these were of the order of one viscous time long, and the flows had been evolved for several viscous times to allow for transients to diminish), of MRW222, MRW212 and T3 at various $R e$. By using a global measure $\left(E_{2}\right)$ rather than a local measure (such as $U$ ), the spectra do not contain any peaks associated with the precession period $T$ (which would introduce a very low frequency peak at $1 / T \sim 10^{-3}$ as well as linear combinations between it and the other main peaks, leading to a very messy spectrum). The $E_{2}$-spectra of MRW222 and MRW212 have a single peak at $2 / T_{m} \approx 0.052$, plus harmonics (below we give an explanation as to why we associate the peak at 0.052 with $T_{m} / 2$ ); this peak is also present in the spectra of T3. At $R e=6850$, the $E_{2}$-spectra of T3 consists of two main peaks, one at $2 / T_{m} \approx 0.052$ and another at $1 / T_{3} \approx 0.008$, plus their linear combinations. At this $R e$, near the onset of $\mathrm{T} 3$, we may regard $\mathrm{T} 3$ as a relative two-torus, i.e. a two-frequency $\left(T_{m}\right.$ and $\left.T_{3}\right)$ quasi-periodic state when viewed in a frame-of-reference rotating with period $T$. 

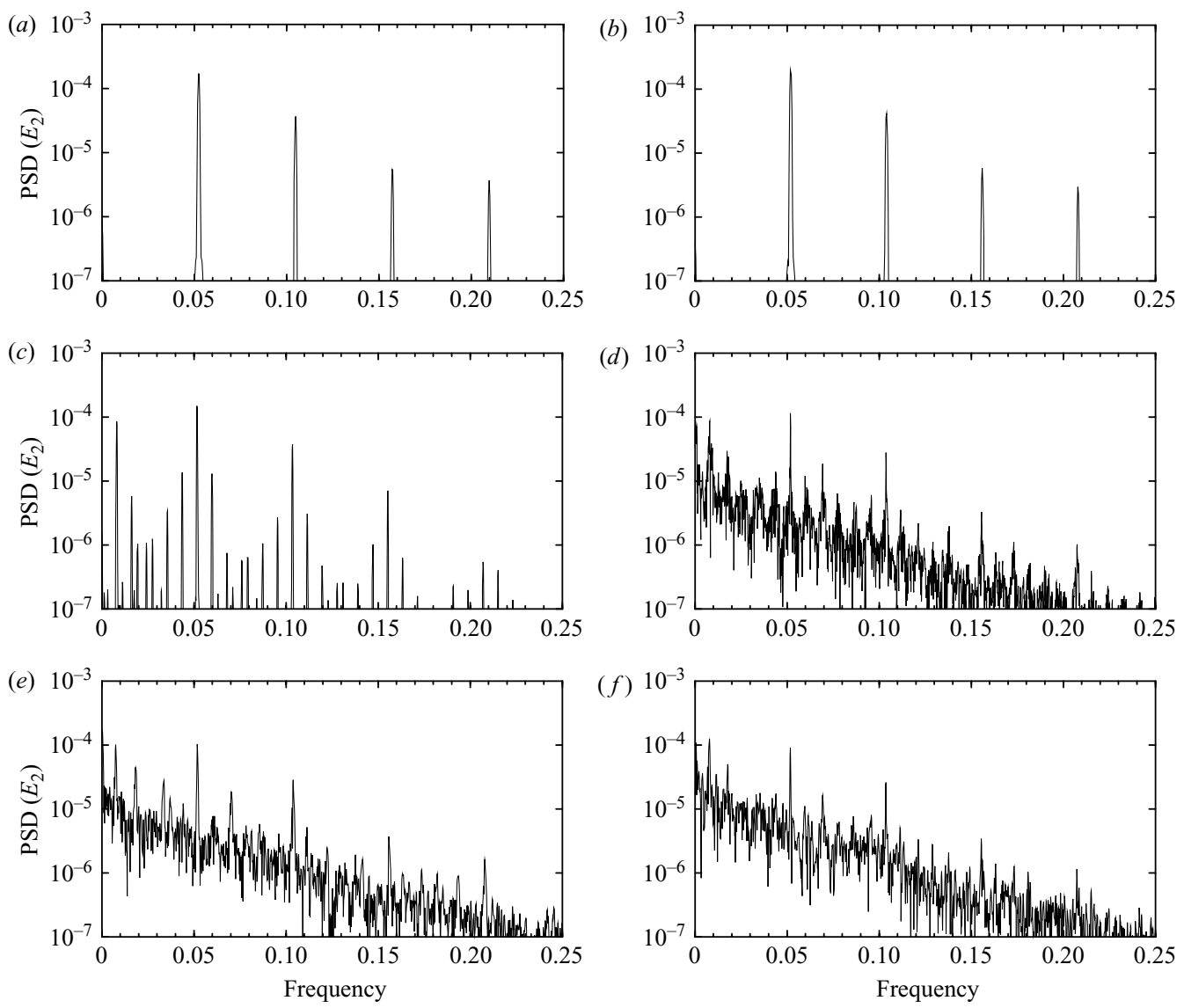

FIgURE 16. Power spectral densities of $E_{2}$ for $(a)$ MRW222 at $R e=6000,(b)$ MRW212 at $R e=6500,(c) \mathrm{T} 3$ at $R e=6850,(d) \mathrm{T} 3$ at $R e=6900$, (e) T3 at $R e=6950$ and $(f) \mathrm{T} 3$ at $R e=7000$.

As $R e$ is increased beyond 6850, the flow becomes more intermittent and the description of $\mathrm{T} 3$ as a relative two-torus appears to be inadequate. The $E_{2}$-spectra at the higher $\operatorname{Re}$ still show peaks at $2 / T_{m}$ and $1 / T_{3}$, but they are quite broadband owing to the intermittent nature of the flow.

Figure 17 shows parts of the time series used to compute the spectra in figure 16 for $\mathrm{T} 3$ at $R e=7000$. The low-frequency modulation that is apparent in the time series of $U$ (it is much more apparent when the time series is several viscous times long) corresponds to the underlying precession period $T \approx 1000$. Figure 8 shows that this period is consistent with the overall variation of $T$ with $R e$ from $R W 2$ through the various modulated rotating waves to T3. From the time series of the modal energies, the $T_{3} \approx 125$ oscillations with the $T_{m} / 2 \approx 19$ modulations riding on top of them are apparent. The $E_{1}$ and $E_{2}$ time series are out of phase at both the $T_{3}$ and $T_{m} / 2$ time scales, and their magnitudes are comparable, indicating significant modal competition leading to the bursting-type dynamics.

Figure 18 shows contours of $w$ at $z=0.8 \Gamma$ for $\mathrm{T} 3$ over approximately one modulation period, $T_{m}$. Note that this figure is not independent of the time origin since the flow is not a relative periodic orbit. Nevertheless, it allows us to make a number of observations. By comparing this figure with figure 14, we see that there 

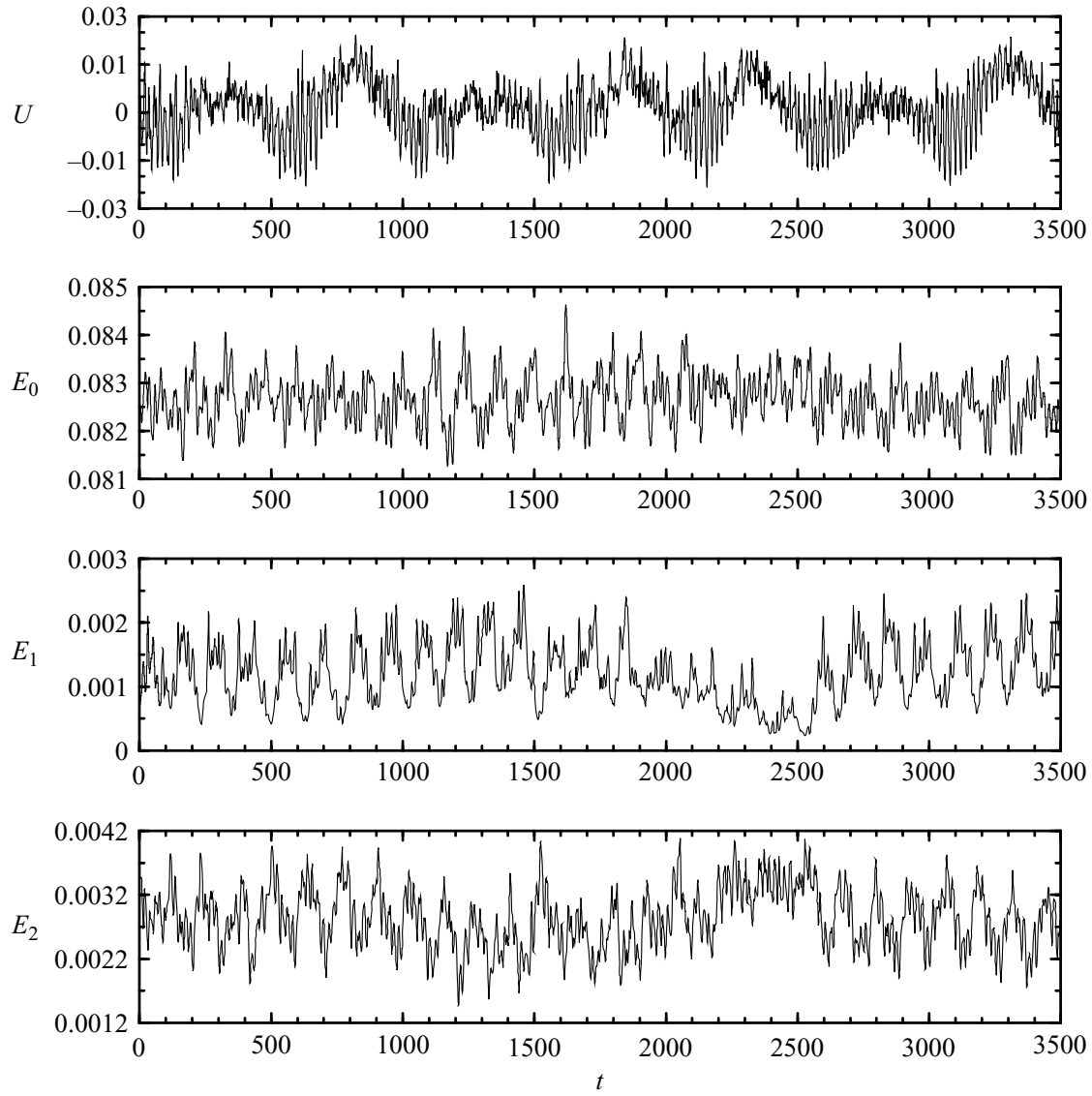

Figure 17. Time series of $U, E_{0}, E_{1}$ and $E_{2}$ for T3 at $R e=7000$.

is a similar precession of the structures at angular rate $\dot{\theta}=2 \pi / T_{m}$. Furthermore, that comparison suggests that the $\mathrm{T} 3$ state, which has strictly broken the spatio-temporal symmetry (3.6) of MRW212, nevertheless shows remnants of it; the contours of T3 at times $T_{m} / 2$ apart are relatively small distortions of each other, whereas for MRW212 they are the same, modulo a small rotation $2 \pi T_{m} / T$. This shows up in the strong peak at frequency $2 / T_{m}$ and its harmonics in the spectra of $\mathrm{T} 3$.

While it is difficult to ascribe a spatial characteristic associated with the new frequency $1 / T_{3}$, it may be associated to the separation of the sidewall boundary layer near the corner $(r=1, z=\Gamma)$, which is becoming more prominent at these higher $R e$. Figure 19 shows contours of T3 in the $\theta=0$ plane at the same times as those in figure 18, where the sidewall boundary-layer separation is evident.

We have seen that the T3 state has broken all spatial and spatio-temporal symmetries, and its temporal characteristics are complex, consisting of aperiodic intermittent bursts. These are characteristics of transitions to so-called weak turbulence - spatio-temporal complexity without very energetic small scales. Figure 20 shows $\left\langle E_{m}\right\rangle$, the modal energies averaged over the precession period $T$, for MRW222, MRW212 and T3 at various $R e$. Since MRW222 has $Z_{2}$ symmetry, only the energy in the even modes are non-zero. MRW212 has broken the $Z_{2}$ symmetry, and so the odd modes are also energetic. The even energies in MRW212 are smaller than those 

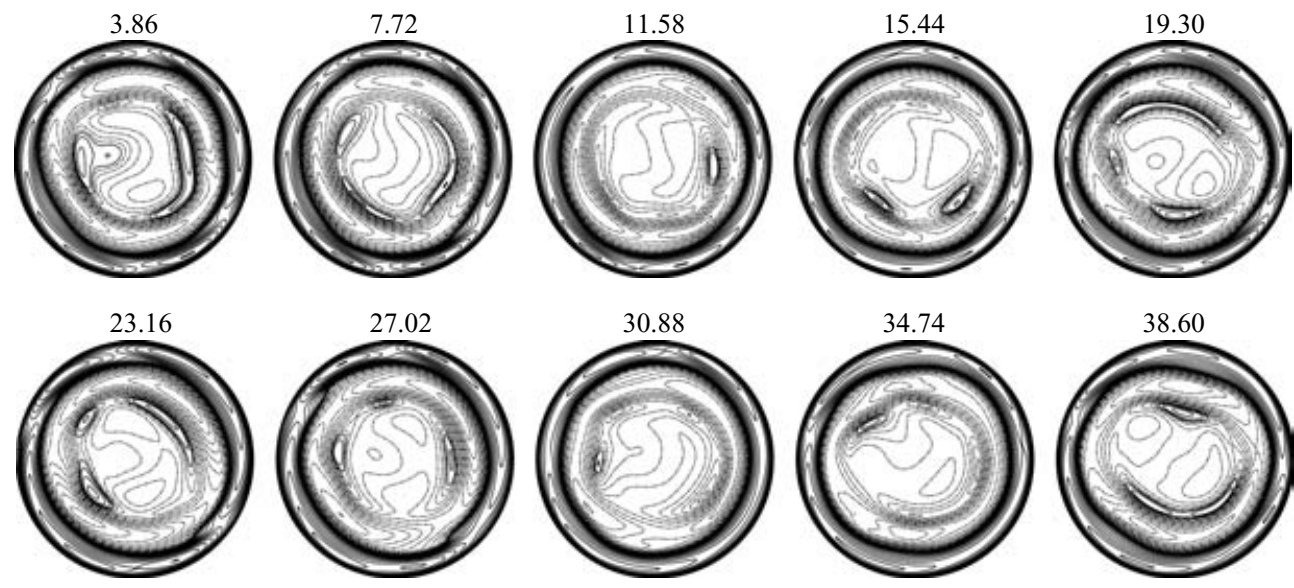

Figure 18. Contours of $w$ at $z=0.8 \Gamma$ at times as indicated over approximately one modulation period, $T_{m}=38.60$, for $\mathrm{T} 3$ at $R e=7000$.
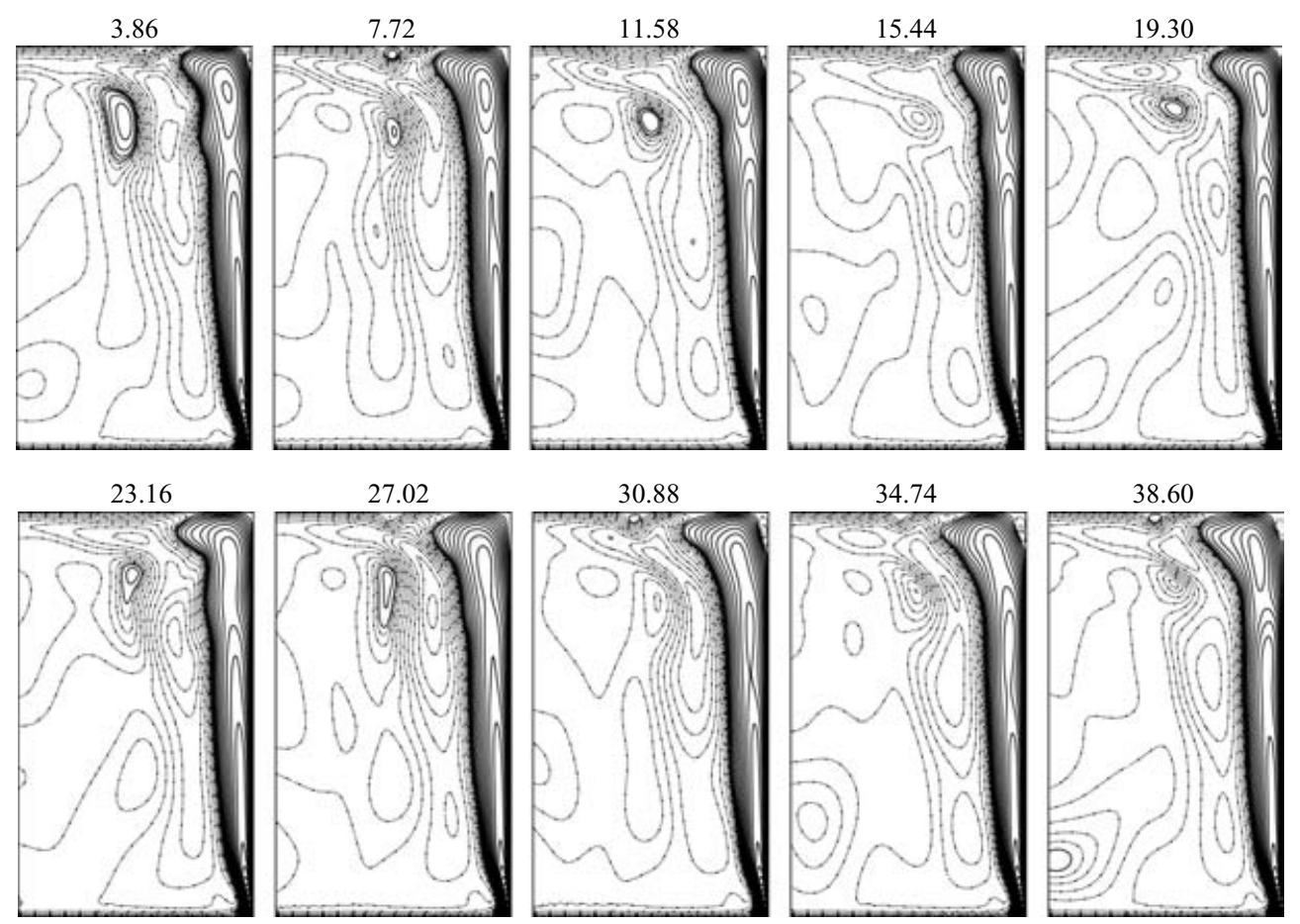

FIGURE 19. Contours of $w$ at $\theta=0$ at times as indicated over approximately one modulation period, $T_{m}=38.60$, for T3 at $R e=7000$.

in MRW222 as a consequence of the growth in the odd energies. For T3, the modal energy spectra is not qualitatively different from that for MRW212; the odd energies have grown, and for small $m$ the even energies have decreased in compensation, but for large $m$ all energies have grown. In all cases, for $m \geqslant 10$ there is a consistent spectral convergence in the tail of the spectra, indicating sufficient numerical resolution. Comparing the contours of T3 at $R e=7000$ in figure 19 with those of RW2 at $R e=4500$ in figure 10 , we see that no new significant small-scale features are evident in $(r, z)$. The smallest scale feature continues to be the boundary layer on the rotating 

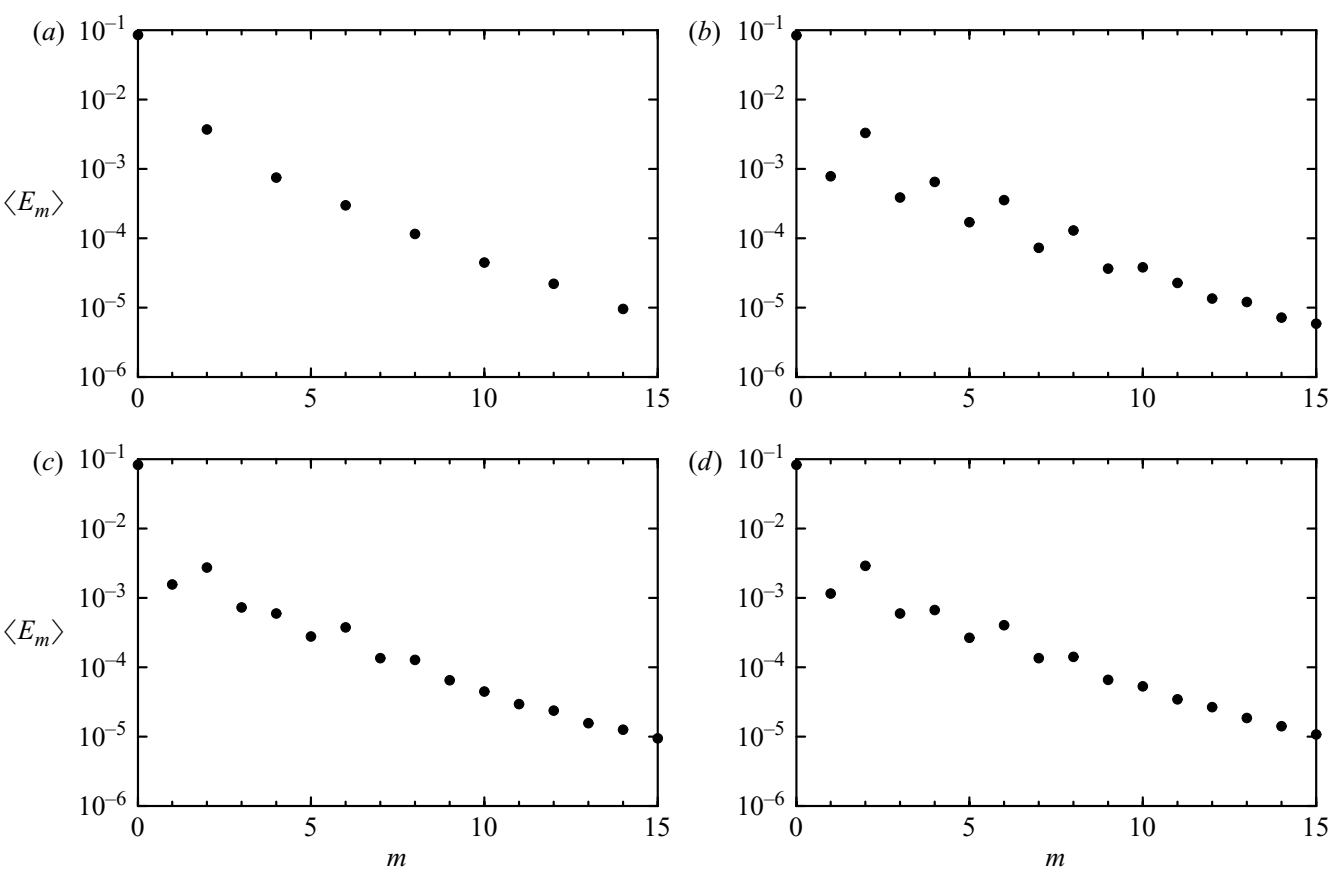

FIGURE 20. Modal energies, averaged over approximated one precession period $T$ for $(a)$ MRW222 at $R e=6000,(b)$ MRW212 at $R e=6500,(c)$ T3 at $R e=6850$ and $(d)$ T3 at $R e=7000$.

endwall, whose thickness scales with $R e^{-0.5}$, and this is well-resolved with the present resolution at the largest $R e=7000$ considered.

\section{Summary and conclusions}

The transition from steady axisymmetric flow to flow with spatio-temporal complexity in an enclosed cylinder driven by the constant rotation of an endwall has been investigated numerically. The system is governed by two parameters, the Reynolds number $R e$ and the aspect ratio $\Gamma$. The investigation has been at fixed $\Gamma=1.72$ where linear stability analysis (Gelfgat et al. 2001) predicts that the first bifurcation of the steady axisymmetric flow preserves the symmetry, and that in the neighbourhood of this value of $\Gamma$, there is competition between two axisymmetric time-periodic modes. Our nonlinear analysis finds both of these axisymmetric limit cycles, LC1 and LC2, bifurcate at about $R e=2660$ (LC1 at $R e=2662.2$ and LC2 at $R e=2665.5$ ) with periods in excellent agreement with the linear stability analysis results. We find both $\mathrm{LC} 1$ and $\mathrm{LC} 2$ to be stable to quite large $R e ; R e \approx 5500$ for LC1 where it becomes unstable to a symmetry-breaking Hopf bifurcation, and $R e \approx 4400$ for LC2 where it suffers a saddle-node bifurcation which is one end of a hysteresis loop with another axisymmetric limit cycle LC3. LC3 is born at a saddle-node bifurcation at $R e \approx 4265$ and continues to be stable to $R e \approx 5840$ where it suffers a period-doubling bifurcation that preserves the axisymmetry, and this period-doubled LC3 is stable to $R e \approx 6050$ where it becomes unstable to a symmetry-breaking Hopf bifurcation.

While all these axisymmetric limit cycle states coexist and are stable to small nonaxisymmetric perturbations, there also coexist a number of stable non-axisymmetric 
states. These non-axisymmetric states can be traced back to $R e \approx 3425$ where a rotating wave RW2 with azimuthal wavenumber $m=2$ is found to be stable for $R e \geqslant 3425$. From a re-examination of the linear stability results of Gelfgat et al. (2001) and the nonlinear results of Marques et al. (2002), it becomes evident that RW2 is the primary mode that first bifurcates from the axisymmetric steady state as $R e$ is increased at lower aspect ratio $(\Gamma<1.63)$. Marques et al. (2002) studied the mode competition between RW2 and LC1 in the neighbourhood of a double-Hopf bifurcation near $\Gamma=1.63$. It is now apparent that the region of coexistence of stable RW2 and stable LC1 extends to $R e>3425$ for $\Gamma=1.72$. Here we have continued the RW2 branch to higher $R e$, following its subsequent bifurcations to modulated rotating waves, and have determined the spatial and spatio-temporal characteristics of these states. All the bifurcations in the transition sequence are in accord with the very general considerations of how symmetries affect the bifurcations of rotating waves (Golubitsky et al. 2000).

For $R e>6050$, the only stable states we have found to survive are the nonaxisymmetric modulated rotating waves, and by $R e \approx 7000$, these have suffered symmetry-breaking bifurcations which result in flows with no spatial or spatiotemporal symmetries and temporal dynamics which consist of three main time scales that differ from one another by about an order of magnitude. The slowest time scale (about $1000 / \Omega$ ) is associated to the precession of the underlying RW2 from which the flow originates, the fastest time scale (about 40/ $\Omega$ ) is associated to the modulation of RW2, and the intermediate time scale (about $125 / \Omega$ ) seems to be associated to the sidewall boundary-layer separation. The flow consists of bursts at the intermediate time scale with the fast modulations dominating in between the bursts, and the overall slow precession superimposed on top of it all. At both the fast and intermediate time scales, the energies in the $m=1$ and $m=2$ azimuthal components of the flow are out of phase, indicating a strong modal competition and exchange of energies leading to the intermittent bursting behaviour.

With the coexistence of multiple axisymmetric and non-axisymmetric states that are stable over an extensive range of $R e$, which one you observe depends on initial conditions. In a perfect experiment, this selection is well-defined in terms of the basins of attraction of the various stable states, but even in the absence of imperfections and noise, the basins can be riddled and their boundaries fractal, and so small differences in initial conditions can result in evolutions to different states. We have not addressed the question of the structure of the basins of attraction in this study. This is a nontrivial exercise for a partial differential equations systems; but it is a very important issue as it gives some indication of the robustness (to noise) of the various stable states, e.g. extraneous noise can drive the system from the basin of one stable state to that of another. If the noise is large, it can actually change the system dynamics completely, e.g. via resonances. These issues warrant further investigation.

This work was performed while visiting the Department of Mechanical Engineering, National University of Singapore. Their hospitality is warmly acknowledged.

\section{REFERENCES}

Barkley, D. \& Henderson, R. D. 1996 Three-dimensional Floquet stability analysis of the wake of a circular cylinder. J. Fluid Mech. 322, 215-241.

Bayliss, A., Matkowsky, B. J. \& Riecke, H. 1994 Structure and dynamics of modulated traveling waves in cellular flames. Physica D 74, 1-23. 
Blackburn, H. M. \& Lopez, J. M. 2000 Symmetry breaking of the flow in a cylinder driven by a rotating end wall. Phys. Fluids 12, 2698-2701.

Blackburn, H. M. \& Lopez, J. M. 2002 Modulated rotating waves in an enclosed swirling flow. J. Fluid Mech. 465, 33-58.

Blackburn, H. M., Marques, F. \& Lopez, J. M. 2005 Symmetry breaking of two-dimensional time-periodic flows. J. Fluid Mech. 522, 395-411.

Brons, M., Voigt, L. K. \& Sorensen, J. N. 1999 Streamline topology of steady axisymmetric vortex breakdown in a cylinder with co- and counter-rotating end-covers. J. Fluid Mech. 401, 275-292.

Chossat, P. \& Iooss, G. 1994 The Couette-Taylor Problem. Springer.

Chossat, P. \& Lauterbach, R. 2000 Methods in Equivariant Bifurcations and Dynamical Systems. World Scientific.

Coles, D. 1965 Transition in circular Couette flow. J. Fluid Mech. 21, 385-425.

Crawford, J. D. \& KNobloch, E. 1991 Symmetry and symmetry-breaking bifurcations in fluid dynamics. Annu. Rev. Fluid Mech. 23, 341-387.

EsCUDIER, M. P. 1984 Observations of the flow produced in a cylindrical container by a rotating endwall. Exps. Fluids 2, 189-196.

Feigenbaum, M. J. 1978 Quantitative universality for a class of nonlinear transformations. J. Stat. Phys. 19, 25.

Gauthier, G., Gondret, P., Moisy, F. \& Rabaud, M. 2002 Instabilities in the flow between coand counter-rotating disks. J. Fluid Mech. 473, 1-21.

Gauthier, G., Gondret, P. \& Rabaud, M. 1999 Axisymmetric propagating vortices in the flow between a stationary and a rotating disk enclosed by a cylinder. J. Fluid Mech. 386, 105-126.

Gelfgat, A. Y., Bar-Yoseph, P. Z. \& Solan, A. 1996 Stability of confined swirling flow with and without vortex breakdown. J. Fluid Mech. 311, 1-36.

Gelfgat, A. Y., Bar-Yoseph, P. Z. \& Solan, A. 2001 Three-dimensional instability of axisymmetric flow in a rotating lid-cylinder enclosure. J. Fluid Mech. 438, 363-377.

Golubitsky, M., LeBlanc, V. G. \& Melbourne, I. 2000 Hopf bifurcation from rotating waves and patterns in physical space. J. Nonlinear Sci. 10, 69-101.

Golubitsky, M. \& Stewart, I. 2002 The Symmetry Perspective: From Equilbrium to Chaos in Phase Space and Physical Space. Birkhäuser.

Golubitsky, M., Stewart, I. \& Schaeffer, D. G. 1988 Singularities and Groups in Bifurcation Theory, Appl. Math. Sci., vol. 2. Springer.

Gorman, M., el Hamdi, M., Pearson, B. \& Robbins, K. A. 1996 Ratcheting motion of concentric rings in cellular flames. Phys. Rev. Lett. 76, 228-231.

Gorman, M., Hamill, C. F., el Hamdi, M. \& Robbins, K. A. 1994 Rotating and modulated rotating states of cellular flames. Combust. Sci. Technol. 98, 25-35.

Gorman, M. \& Swinney, H. L. 1982 Spatial and temporal characteristics of modulated waves in the circular Couette system. J. Fluid Mech. 117, 123-142.

Gorman, M., Swinney, H. L. \& Rand, D. A. 1982 Doubly periodic circular Couette flow: experiments compared with predictions from dynamics and symmetry. Phys. Rev. Lett. 46, 992-995.

Guckenheimer, J. \& Holmes, P. 1997 Nonlinear Oscillations, Dynamical Systems, and Bifurcations of Vector Fields. Springer.

Hide, R. \& Titman, C. W. 1967 Detached shear layers in a rotating fluid. J. Fluid Mech. 29, 39-60.

Iooss, G. \& Adelmeyer, M. 1998 Topics in Bifurcation Theory and Applications, 2nd edn. World Scientific.

Knobloch, E. 1994 Bifurcations in rotating systems. In Lectures on Solar and Planetary Dynamos (ed. M. R. E. Proctor \& A. D. Gilbert), pp. 331-372. Cambridge University Press.

KNOBLOCH, E. 1996 Symmetry and instability in rotating hydrodynamic and magnetohydrodynamic flows. Phys. Fluids 8, 1446-1454.

Krupa, M. 1990 Bifurcations of relative equilibria. SIAM J. Math. Anal. 21, 1453-1486.

KuzNetsov, Y. A. 1998 Elements of Applied Bifurcation Theory, 2nd edn. Springer.

Lopez, J. M. 1990 Axisymmetric vortex breakdown. Part 1. Confined swirling flow. J. Fluid Mech. 221, 533-552.

Lopez, J. M. 1998 Characteristics of endwall and sidewall boundary layers in a rotating cylinder with a differentially rotating endwall. J. Fluid Mech. 359, 49-79. 
Lopez, J. M., Hart, J. E., Marques, F., Kittelman, S. \& Shen, J. $2002 a$ Instability and mode interactions in a differentially-driven rotating cylinder. J. Fluid Mech. 462, 383-409.

Lopez, J. M. \& MARQues, F. 2004 Mode competition between rotating waves in a swirling flow with reflection symmetry. J. Fluid Mech. 507, 265-288.

Lopez, J. M., Marques, F. \& SANChez, J. 2001 Oscillatory modes in an enclosed swirling flow. J. Fluid Mech. 439, 109-129.

Lopez, J. M., MARques, F. \& SHEN, J. $2002 b$ An efficient spectral-projection method for the NavierStokes equations in cylindrical geometries II. Three dimensional cases. J. Comput. Phys. 176, 384-401.

Lopez, J. M. \& Perry, A. D. 1992 Axisymmetric vortex breakdown. Part 3. Onset of periodic flow and chaotic advection. J. Fluid Mech. 234, 449-471.

Lopez, J. M. \& SHEN, J. 1998 An efficient spectral-projection method for the Navier-Stokes equations in cylindrical geometries I. Axisymmetric cases. J. Comput. Phys. 139, 308-326.

Lugt, H. J. \& Aвboud, M. 1987 Axisymmetric vortex breakdown with and without temperature effects in a container with a rotating lid. J. Fluid Mech. 179, 179-200.

Marques, F. \& Lopez, J. M. 2001 Precessing vortex breakdown mode in an enclosed cylinder flow. Phys. Fluids 13, 1679-1682.

Marques, F., Lopez, J. M. \& Blackburn, H. M. 2004 Bifurcations in systems with $Z_{2}$ spatiotemporal and $O(2)$ spatial symmetry. Physica D 189, 247-276.

Marques, F., Lopez, J. M. \& Shen, J. 2002 Mode interactions in an enclosed swirling flow: a double Hopf bifurcation between azimuthal wavenumbers 0 and 2. J. Fluid Mech. 455, 263-281.

Moisy, F., Pasutto, T. \& Rabaud, M. 2003 Instability patterns between counter-rotating disks. Nonlinear Process. Geophys. 10, 281-288.

Mullin, T. 1999 Flow state multiplicity in convection. Phys. Fluids 11, 2815-2817.

Newhouse, S., Ruelle, D. \& TAKens, F. 1978 Occurrence of strange axiom-A attractors near quasi-periodic flows on $T^{m}, m \geqslant 3$. Commun. Math. Phys. 64, 35.

Nore, C., Moisy, F. \& Quartier, L. 2005 Experimental observation of near-heteroclinic cycles in the von Kármán swirling flow. Phys. Fluids 17, 064103.

Nore, C., Tuckerman, L. S., Daube, O. \& Xin, S. 2003 The 1:2 mode interaction in exactly counter-rotating von Kármán swirling flow. J. Fluid Mech. 477, 51-88.

RAND, D. 1982 Dynamics and symmetry. Predictions for modulated waves in rotating fluids. Arch. Rat. Mech. Anal. 79, 1-38.

READ, P. L. 2001 Transition to geostrophic turbulence in the laboratory, and as a paradigm in atmospheres and oceans. Surv. in Geophys. 22, 265-317.

Renardy, M. 1982 Bifurcation from rotating waves. Arch. Rat. Mech. Anal. 79, 49-84.

Ruelle, D. \& Takens, F. 1971 On the nature of turbulence. Commun. Math. Phys. 20, 167.

Schouveiler, L., Gal, P. L., Chauve, M. P. \& Takeda, Y. 1999 Spiral and circular waves in the flow between a rotating and a stationary disc. Exps. Fluids 26, 179-187.

Serre, E. \& Bontoux, P. 2002 Vortex breakdown in a three-dimensional swirling flow. J. Fluid Mech. 459, 347-370.

Serre, E., Tuliszka-Szzitko, E. \& Bontoux, P. 2004 Coupled numerical and theoretical study of the flow transition between a rotating and a stationary disk. Phys. Fluids 16, 688-706.

Sorensen, J. N. \& Christensen, E. A. 1995 Direct numerical simulation of rotating fluid flow in a closed cylinder. Phys. Fluids 7, 764-778.

Sotiropoulos, F. \& Ventikos, Y. 2001 The three-dimensional structure of confined swirling flows with vortex breakdown. J. Fluid Mech. 426, 155-175.

Sotiropoulos, F., Ventikos, Y. \& LaCkey, T. C. 2001 Chaotic advection in a three-dimensional stationary vortex-breakdown bubbles: Šil'nikov's chaos and the devil's staircase. J. Fluid Mech. 444, 257-297.

Spohn, A., Mory, M. \& Hopfinger, E. J. 1998 Experiments on vortex breakdown in a confined flow generated by a rotating disk. J. Fluid Mech. 370, 73-99.

Stevens, J. L., Lopez, J. M. \& CANTwell, B. J. 1999 Oscillatory flow states in an enclosed cylinder with a rotating endwall. J. Fluid Mech. 389, 101-118.

Swift, J. W. \& WiESENFELD, K. 1984 Suppression of period doubling in symmetric systems. Phys. Rev. Lett. 52, 705-708.

VENTIKos, Y. 2002 The effect of imperfections on the emergence of three-dimensionality in stationary vortex breakdown bubbles. Phys. Fluids 14, 13-16. 